\title{
Les emplois linguistiques et culturels derrière les textes aljamiados
}

\author{
Nuria de Castilla \\ EPHE, PSL, Paris \\ nuria.decastilla@ephe.sorbonne.fr
}

Les manuscrits complets produits, copiés et/ou transmis par les Mudéjares et les Morisques, de même que les fragments et documents qui ont été employés dans les ais de reliures qui leur ont appartenu, constituent des témoins uniques pour nous permettre d'étudier l' ample éventail linguistique et culturel de ces communautés ${ }^{1}$. Nous possédons une vingtaine de documents et environ deux cents manuscrits écrits principalement sur du papier, mais également sur du parchemin; il existe des copies de grand format et d'autres très petites, des livres très volumineux et des documents d'un seul feuillet; ils peuvent être en une seule langue ou dans plusieurs - principalement arabe et castillan (dans différents stades linguistiques), mais aussi catalan et latin, les uns et les autres transcrits dans trois alphabets différents: arabe, latin et hébreu²

Pour bien comprendre cette production écrite, il faudrait prendre en compte les facteurs qui régissent tout acte de communication: émetteur, destinataire, voie, code, message et contexte. Pour parler de la littérature morisco-aljamiado, cette façon de procéder a été simplifiée et on a tendu à identifier le «code» employé par les Mudéjares et les Morisques avec le castillan en caractères arabes en oubliant les autres composants de la transmission.

Cette absence de répertoire de données objectives et d' analyse linguistique en relation avec le contexte nous offre des résultats qui peuvent conduire à des erreurs ${ }^{3}$. Seule une perspective plus large, mettant en rapport la langue

1 Martínez de Castilla, «El libro manuscrito».

2 L'alphabet hébreu est associé uniquement à des documents en judéo-espagnol ou en hébreu aljamiado réemployés dans les ais d'un manuscrit d'Almonacid de la Sierra (Aragon). Voir $\S$ 1.1.4.

3 Les éditions de textes écrits (ou au moins copiés) en arabe au sein de ces communautés, de même que le développement de recherches à leur sujet, sont pratiquement inexistants. De fait, on relèvera que des passages en arabe ont été complètement omis dans des thèses docto- 
et l'alphabet employés dans chaque cas avec la matérialité du codex (code et voie) et, à son tour, avec les contenus du texte (message), pourra nous conduire à avancer une hypothèse sur l'émetteur (copiste) et le destinataire (lecteur ou auditeur). Tout cela nous aidera ensuite à comprendre le contexte dans lequel ces textes ont été produits et nous permettra de voir que, à travers les usages linguistiques que présentent un bon nombre de leurs manuscrits, la forte acculturation et islamisation ${ }^{4}$ que l' on associe à ces communautés ${ }^{5}$ semblent se heurter à des arguments de poids véhiculés par les textes que les Mudéjares et les Morisques eux-mêmes possédaient, traduisaient, copiaient, lisaient et entendaient.

Dans le présent article, je me limiterai à présenter les usages linguistiques et culturels de l'aljamia dans son contexte historique, linguistique, matériel et culturel ${ }^{6}$.

L'aljamia est un concept très difficile à définir étant donné qu'il ne s'agit pas à proprement parler d'une langue, ni d'un type d'écriture, ni de contenus spécifiques. Lorsque nous parlons de littérature aljamiada, nous voulons parler, de façon générale, des textes copiés et produits par des Mudéjares et des Morisques, dans lesquels ils employaient une variante linguistique du castillan, caractérisée par une forte influence de l'arabe et de l'aragonais et par son archaïsme, qu'ils écrivaient en caractères arabes. Ces textes furent trans-

rales portant sur des manuscrits morisques, comme cela est le cas dans l' ouvrage de Sánchez Álvarez, El manuscrito misceláneo, où on ne trouve pas même une référence aux cent feuillets de texte coranique en arabe qui figurent dans le manuscrit; l'édition du manuscrit Paris, BnF Arabe 1163 , qui a fait l' objet de la thèse de Suárez Piñera, Publicación y estudios, prend seulement en compte les passages aljamiados (mais dans ce cas, le titre l'indique).

4 Voir, par exemple, les titres de quelques articles influencés par la proposition de Hegyi pendant le premier congrès sur l'aljamiado organisé par Galmés de Fuentes en 1987: «Una variante islámica del español»: Sánchez Álvarez, «Sobre la variante islámica» ou Gómez Renau, «La lengua aljamiada [...]: una variante islámica».

5 Domínguez Ortiz et Vincent, Historia de los moriscos.

6 J'aborde ce projet en étant consciente de la difficulté qu' il y a à définir le concept d' aljamia à supposer que cela soit possible. Pour cette raison, il est compréhensible que les organisateurs de l' atelier sur Writing systems of Garshuni, Judeo-Arabic and Samaritan-Arabic, outre les sections «Ottoman», «Judéo-arabe», «Garshuni», «Samaritain», «Copte-arabe», aient choisi «Espagne» pour celle consacrée à l' aljamia; peut-être n'y avait-il pas d' autre façon de désigner ce sujet. Princeton, Institute for Advanced Study, organisé par Sabine Schmidtke et George Kiraz, 9-10 juin 2016. 
crits entre la fin du XIV siècle et le début du XVII ${ }^{\mathrm{e}}$, particulièrement dans les royaumes d'Aragon et de Castille, mais aussi dans d' autres endroits, principalement en Afrique du Nord où de nombreux exilés morisques trouvèrent refuge. Toutefois, nous verrons au long de cet article que cette définition peut sur chacun de ses points trouver un contre-exemple; et si cela rend parfois le concept dont nous souhaitons traiter difficile à cerner, c' est également une preuve de la richesse et de la complexité de ce phénomène.

De nosjours, selon le dictionnaire de la Real Academia Española ${ }^{7}$, «aljamia» peut avoir trois sens:1) «Parmi les anciens habitants musulmans de l'Espagne, langue des chrétiens de la péninsule»; 2) «Un texte morisque en langue romane, mais transcrit en caractères arabes » et 3) «Texte judéo-espagnol transcrit en caractères hébreux». Je me concentrerai sur les deux premiers.

1.1 Langues (voie)

1.1.1 Castillan

1) «Parmi les anciens habitants musulmans de l' Espagne, langue des chrétiens de la péninsule.» Nous savons que l'emploi du mot «aljamía» comme synonyme de «castillan » était très répandu dès le $\mathrm{xv}^{\mathrm{e}}$ siècle:

El cerro de Caxantepenin, que quiere decir en aljamía «cerro de piedras» $(1493)^{8}$

Lo tercero, que no hablasen algarabía, sino que todos hablasen en aljamía, y que todas las escrituras y contratos que se solían hacer en arábigo se hiciesen en castellano (1604-1618) .

Ocultamente traía consigo una cierta moneda de plata en que había en ella letras y caracteres en lengua arábiga, que decían «ley lehe ele Alá», que [en] aljamía querían decir que no hay otro señor sino Dios solo (Granada, 1574$)^{10}$.

7 Edition électronique du dictionnaire (www.rae.es), consulté le 12 décembre 2016, s.v. «aljamía».

8 «Carta autorizada por Bernardina de Madrid» [Documentos históricos de Málaga], [CORDE], consulté le 20 décembre 2016.

9 Fray Prudencio de Sandoval, Historia de la vida y hechos del emperador Carlos V, 1604-1618, CORDE, consulté le 20 décembre 2016.

10 Archivo Diocesano de Cuenca, Leg. 262, núm. 3.573, CORDE, consulté le 20 décembre 2016. Pour plus d'exemples et de sens du mot «aljamia», voir Martínez de Castilla, «Anduve mirando», pp. 235-246. 
Mais outre le castillan, les chrétiens employaient d'autres langues qui sont rarement citées dans les études sur ce type de littérature ou encore dans les définitions qui sont données aux $\mathrm{XV}^{\mathrm{e}}$ et $\mathrm{XVI}^{\mathrm{e}}$ siècles. Cela était peut-être dû au fait qu' elles n'étaient pas écrites en caractères arabes (c' est le cas du catalan dans le Llibre de la çuna exara §3.2.1) ou qu' elles n'étaient pas employées dans un contexte strictement lié au milieu religieux musulman (c'est le cas du latin). Il s' agit cependant de langues que nous trouvons également dans les textes mudéjares et morisques.

\subsubsection{Catalan $(\S 3.2 .1)$}

A la fin du XV $\mathrm{XV}^{\mathrm{e}}{ }^{11}$, à Valence, un cadi (ou un de ses proches), en d' autres termes un Mudéjar, rédigea en catalan un traité juridique musulman qui portait le titre de Llibre de la çunna e xara. Le destinataire de ce texte écrit en caractères latins était un noble chrétien ${ }^{12}$.

\subsubsection{Latin}

Aux $\mathrm{XV}^{\mathrm{e}}$ et $\mathrm{XVI}^{\mathrm{e}}$ s., le latin est la langue employée par les érudits et les ecclésiastiques. En dépit de l'usage limité de cette langue par le reste de la population, les écrits morisques contiennent des passages en latin, même si c'est de manière exceptionnelle. Ils sont bien entendu dans leur majorité liés aux prières chrétiennes que les Morisques devaient réciter au cours du processus de catéchisation consécutif à leur conversion obligatoire en 1502 en Castille ou en $15^{26}$ en Aragon $(\S 1.3 .1)^{13}$. Il existe cependant d' autres cas dans lesquels nous rencontrons cette langue, par exemple pour les termes de botanique en latin insérés dans des textes en aljamia ${ }^{14}$, ou dans les rares textes médicaux qui figurent dans cette production ${ }^{15}$ et dans lesquels le latin est employé parmi les langues de base du manuscrit. Bien qu'il n'en aille pas ainsi dans le premier cas, le fait que le latin soit écrit en caractères latins semble indiquer que tant le copiste que le récepteur n' avaient pas de problèmes pour le comprendre $(\S 1 \cdot 3 \cdot 3)$.

Dans cette même situation de compréhension du latin, probablement, il existe une série de documents dans cette langue qui ont été produits dans l'Espagne chrétienne médiévale et qui ont été connus et utilisés par les Mudé-

\footnotetext{
11 À propos de la différence entre la date du manuscrit et celle proposée par l'éditrice, voir Barceló, Un tratado catalán, p. XIV.

12 Barceló, Un tratado catalán, p. XIv. Voir §3.2.1.

13 Domínguez Ortiz et Vincent, Historia de los moriscos, p. 96.

14 Comme dans Madrid, BRAH 11/9415. Voir Martínez de Castilla, Una biblioteca, chap. III.

15 Práctica [de medicina], Madrid, BRAH 11/9411.
} 


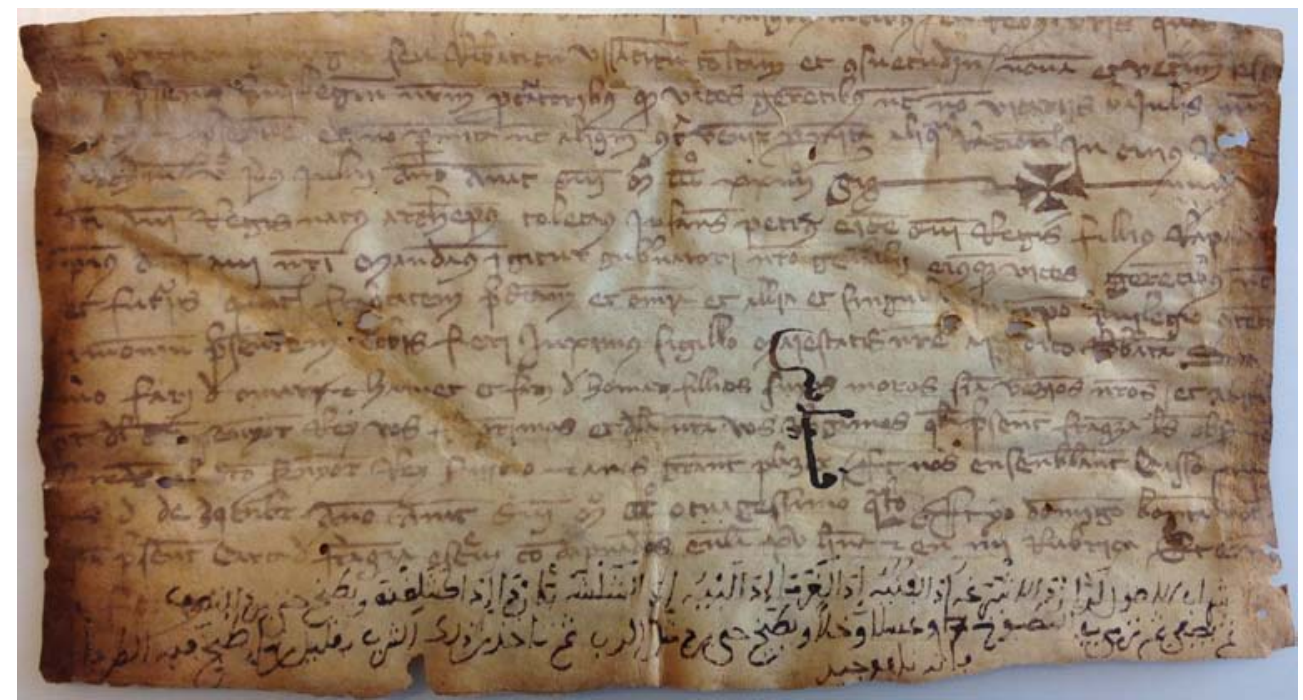

FIGURE 1 Madrid, CCHS-CSIC, TNT RESC/98.4.a. Parchemin avec un texte arabe ajouté à une date ultérieure sous le texte latin (C)CSIC, CCHS, BIBLIOTECA TNT

jares et les Morisques. Nous avons la certitude que ces documents étaient en circulation parmi eux en raison du témoignage à ce propos que nous trouvons parmi les papiers et les manuscrits de la collection d'Almonacid de la Sierra (de nos jours au CCHS-CSIC) où des annotations en arabe ont été ajoutées (fig. 1 $)^{16}$. Bien que de façon limitée, la présence d'écrits et de documents en latin dans ces communautés invite à une nouvelle lecture sur la connaissance et l'usage de cette langue par certains membres de la communauté morisque; cela suppose de nouvelles hypothèses sur le degré de formation de certains d'entre eux.

\subsubsection{Arabe}

Il est certain que l'arabe n'était pas une langue employée habituellement par les chrétiens aux XVe et XVI ${ }^{\mathrm{e}}$ s. $^{17}$, mais les Mudéjares et les Morisques l'utilisaient beaucoup dans leurs écrits, et ce bien que l'idée selon laquelle les Morisques hispanophones «avaient oublié l'arabe» soit constamment re-

16 Sur la découverte, voir Martínez de Castilla, «Qur’anic manuscripts», pp. 89-9o et la bibliographie citée dans cet article.

17 Voir García-Arenal et Rodríguez Mediano, Un Oriente español; Martínez de Castilla, «The Teaching and Learning of Arabic». 
prise $^{18}$. Une étude sur les manuscrits trouvés à Almonacid de la Sierra offre un résultat éloquent: $37,5 \%$ des manuscrits qui furent découverts cachés dans une maison de ce bourg d'Aragon étaient écrits en langue arabe, 62,5\% employant l'aljamia ${ }^{19}$. En dépit de l'importance de l'arabe dans la culture mudéjare et morisque ${ }^{20}$, les écrits de ces communautés sont généralement associés à l'aljamia, habituellement définie comme du castillan écrit en caractères arabes et dans lequel est utilisé un pourcentage élevé de termes arabes, d'aragonismes et d' archaïsmes (voir $§ 1.2$ et 1.3).

\section{$1.2 \quad$ Etats linguistiques du castillan}

En dépit de la forte et constante équivalence entre les mots «aljamia» et «castillan» depuis le $\mathrm{XV}^{\mathrm{e}}$ s., on trouve dans les textes qui faisaient partie des collections mudéjares et morisques de nombreux écrits qui présentent un état de la langue différent de celui de l' espagnol standard des $\mathrm{XV}^{\mathrm{e}}$ et XVI $\mathrm{X}^{\mathrm{e}} \mathrm{s}$., autant en caractères arabes que latins. Ils coexistent avec des textes standardisés pour lesquels, également, les deux alphabets sont utilisés.

1.2.1 Variante linguistique «islamisée »

Les textes qui présentent un état de langue différent de l' espagnol standard se caractérisent par la forte empreinte de l'arabe et de l'aragonais qu' ils conservent et par leur caractère archaïsant.

Biçmi Illāhi i'rraḥmāni i'rrahīm. Fue recontado que Omar ibnu Alhatab, radīya Allahu canhu, rogó ada Allah que le diese a ver los arruhes de los muertos en su dormir. Y llevó Allah su arruh a un almacábir y vido los muertos asentados de cara dell alquibla de sus fuesas, unos con otros a corros. Y dio Omar açalam sobre ellos y no tornaron sobr'él el açalam²1.

Quoique cette variante linguistique du castillan soit propre aux Mudéjares et aux Morisques, nous ne pouvons pas dire qu' elle ait été la langue qui les dis-

18 Galmés de Fuentes, «La lengua española», p. 21.

19 Zanón, «Los estudios de lengua árabe».

20 Pas seulement pour les originaux d' où dérivent les traductions morisques, comme l' a noté Wiegers («Datation», p. 30), mais aussi dans l'utilisation même de l'arabe par ces communautés.

21 Madrid, BNE MSs/4953, ff. $77^{\mathrm{r}}-78^{\mathrm{r}}$. Edité par Martínez de Castilla, El manuscrito aljamiado T19, pp. 513-514. Je normalise la transcription en suivant les mêmes critères que les Morisques quand ils écrivaient en caractères latins; je respecte fidèlement le texte arabe des doxologies dont la translittération apparaît en italiques. 
tinguait puisqu' aussi bien ils employaient l' espagnol standard. La raison d' être de cette variante - communément dite «islamisée»-22 ne semble pas reposer sur le fait que l'auteur du texte ait été musulman ou morisque, qu'il ait employé tel ou tel type d'écriture (caractères arabes ou latins, §1.3) ou que ses textes aient eu telle nature ou tel contenu. Les écrits les plus arabisés et archaïsants, semble-t-il, sont directement en rapport avec leur origine linguistique: il s' agit de chapitres ou de livres de sujets différents, mais toujours traduits de l'arabe. Dans toutes ces traductions, on trouve ces traits arabisants et archaïsants en proportion plus ou moins grande selon deux facteurs fondamentaux: la date à laquelle la traduction a été réalisée (une donnée objective qui nous échappe dans la plupart des cas) et, bien sûr, l'état du castillan à ce moment de même que les compétences linguistiques du traducteur ${ }^{23}$. Dans une seconde étape, qui coïncide avec la transcription de ces traductions, deux aspects fondamentaux interviennent qui peuvent expliquer les différences dans la proportion de traits spécifiques de cette variante linguistique: le caractère innovateur ou conservateur du copiste (ce qui le conduira ou non à introduire des nouveautés au modèle qu'il copie) de même que sa connaissance des deux langues (ce qui lui permettra de mieux comprendre et d' exprimer avec une plus grande correction les sens possibles de tel ou tel passage).

Afin de comprendre cette variante du castillan si répandue dans les communautés musulmanes qui continuaient à vivre en Espagne au XVI ${ }^{\mathrm{e}} \mathrm{s}$. et au début du XVII ${ }^{\mathrm{e}}$, les spécialistes ont tenté de mettre en place des catégories de différentes natures: «variedad romance de las comunidades islámicas hispánicas durante la Edad Media y el Siglo de Oro ${ }^{24}$; subdialecte ou idiolecte ${ }^{25}$; sociolecte, ethnolecte ou hiérolecte ${ }^{26}$, etc. Sans qu'il ait été possible de parvenir à une conclusion nette, l' argument avancé le plus souvent pour expliquer ces caractéristiques linguistiques est le caractère isolé des communautés morisques qui restaient à l'écart des courants linguistiques de l'époque ${ }^{27}$; cet

22 Voir les titres de certains articles influencés par la proposition de Hegyi lors du premier congrès organisé par Galmés de Fuentes en 1987, cités dans la n. 4 de cet article.

23 Montaner, «La aljamía», et Martínez de Castilla, Una biblioteca morisca, chap. III. Il est très important de noter que des mots sont des archaïsmes selon les époques.

24 Montaner, «Literatura aljamiada», p. 46.

25 Martínez de Castilla, Una biblioteca morisca, chap. III.

26 Brisville-Fertin, «El aljamiado como discurso de una comunidad. Los nuevos campos lingüísticos en el estudio de los textos moriscos ». Communication presentée lors du colloque La voz oculta: en torno a los textos aljamiados, Madrid, 2012. Je remercie Olivier BrisvilleFertin pour l' aimable envoi de son texte.

27 Hegyi, «Una variante», p. 652; Harvey, «El Mancebo de Arévalo», p. 23. 
argument cesse toutefois d'avoir du poids si l'on prend en compte les textes écrits par des Mudéjares et des Morisques qui employaient le castillan standard.

1.2.2 Castillan standard, avec influence de l' aragonais

A côté de la variante linguistique arabisée et archaïque du castillan, nous possédons des textes dans lesquels on emploie l' espagnol standard: il s'agit de compositions nouvelles ou d' origine européenne, et non de traductions ${ }^{28}$. Le texte en caractères latins qui a été ultérieurement appelé Tratado de los dos caminos est un exemple représentatif des premières:

De lo que queda de la verdad é sacado este libro, y aunque en él halles algunas cosas de entretenimiento, solo es para mostrarte el camino que llevan los que ciegamente van a parar // a la cueva escura del infierno. [...] Mi intención es apartarte d' ellas, y ser causa de que vayas por el camino dichoso $^{29}$.

C' est également le cas des textes du Mancebo de Arévalo, en caractères arabes cette fois ${ }^{30}$ :

Y yo comencé esta obra ocho años después de la dicha conversión, con ayuda de un escolano de buena doctirina, avisado y de largo šarḥe. Era natural de Arévalo, y dezía que su madre fue cristiana veinticinco años. Y con su ayuda, hize lo más d'esta copilación, a loor y gracia de nuestro grande y verdadero Allah, señalando algunas aleyas de nuestro // onrado Alcorán, de donde se tomó lo más d' este conpendio ${ }^{31}$.

S' il est vrai que, dans le texte du Mancebo qui a été cité figure un terme arabe, et ce en raison de sa nature même, cela disparaît dans les translittérations en caractères arabes de textes littéraires qui circulaient en castillan dans les communautés chrétiennes et qui plaisaient aussi aux Morisques. Il en va ainsi, par exemple, des Amores de Parísy Diana ou des Dichos de los siete sabios de Grecia:

28 Bien qu'il s'agisse d'une différence linguistique très notable, elle n'a pas reçu toute l'attention qu' elle mérite.

29 Madrid, BRAH 11/9394, ff. $2^{\mathrm{r}-\mathrm{v}}$. Apud Galmés de Fuentes (éd), Tratado de los dos caminos, p. 193. Je normalise la graphie du texte édité.

30 Pour une réflexion sur l' emploi de l' expression «aljamia en caracteres arabes o latinos» et une nouvelle proposition, voir Casassas, «La literatura aljamiado-morisca».

31 Cambridge, UL, Dd. 9.49, ff. $4^{\mathrm{v}}-5^{\mathrm{r}}$. Apud Harvey, «Un manuscrito aljamiado», p. 199. Je normalise la graphie du texte édité. 
Lo primero que harás:

onrarás aquel / Dios solo, que gobierna tierra y polo

Trabaja bien de contino por todas / partes y modos;

da siempre plaze $/ \mathrm{r}$ a todos ${ }^{32}$.

Les traducteurs et/ou copistes introduisaient consciemment ou non leurs propres variantes locales ce qui explique que, comme la production morisque était principalement aragonaise, il est normal de trouver une forte proportion d'aragonismes dans ces textes ${ }^{33}$. Tout dépend, une fois de plus, des compétences linguistiques du copiste, de son côté conservateur ou innovateur, de même que de l'époque à laquelle le texte a été copié ou composé; en principe, plus la date est tardive, plus il est castillanisé ${ }^{34}$.

\section{$1.3 \quad$ Alphabet (code)}

La partie la plus facile de la définition de la Real Academia Española semble la dernière: «transcrit en caractères arabes ». Cela pose toutefois un problème vu qu' au sein de ces communautés nous trouvons aussi bien le castillan «islamisé » - que je préfère qualifier d' arabisé - que le castillan standard transcrit en caractères arabes et latins.

\subsubsection{Caractères arabes}

Selon la définition, un texte aljamiado est un texte en langue romane [= castillan] en caractères arabes. De fait, la majorité des textes produits et copiés par des Mudéjares et des Morisques présentent cette caractéristique, mais pas tous.

Comment interpréter des mots comme هتل (《hôtel»)? Nous ne parlons pas d'aljamia, mais d'emprunt linguistique. La même chose se produit quand on écrit des noms castillans en caractères arabes. C'est le cas, par exemple, de la signature arabe du roi d'Aragon Pedro I ${ }^{\text {er }}$ (vers 1068-1104) (fig. 2).

Selon Montaner ${ }^{35}$, «en árabe andalusí se leería "ráš(a)m Béțro Péțro (a)ben Šánğo Šánčo" = 'señal de Pedro, hijo de Sancho'». Il ajoute que Francisco

32 Galmés de Fuentes (éd), Dichos de los siete, p. 47.

33 Parallèlement, le «seseo» apparaît dans Madrid, BNE Mss/6016, et a été expliqué par une possible origine andalouse du copiste. Edité par Liman, Edición crítica.

34 Martínez de Castilla, Una biblioteca, p. 145.

35 Montaner, «La Historia Roderici», p. 51, n. 219. 


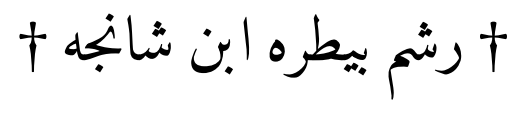

FIGURE 2

Rašam Pețro ibn Sancho («Signature de Pedro fils de Sancho»)

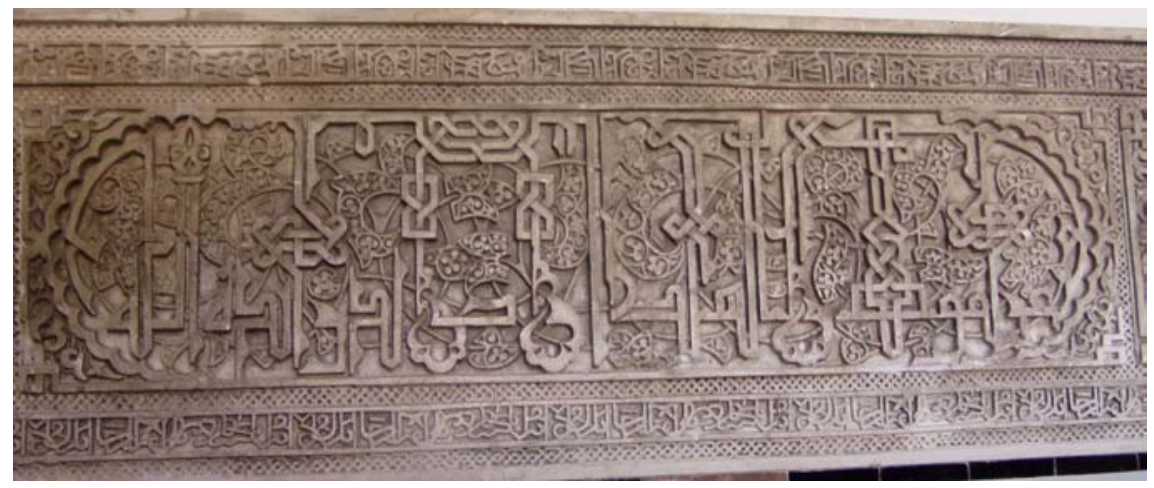

FIgURE 3 Allusion au sultan Don Pedro ضن بدر. Vestibule du palais mudéjar du roi Don Pedro, Alcazar de Séville

J. Hernández, dans son travail sur cette signature, explique de manière erronée que le nom est «escrito en aljamiado ${ }^{36}$. Il semble cependant que le nom en lui-même n' est écrit ni en aljamia, ni en arabe, mais qu'il s'agit d'une stricte transcription du castillan en caractères arabes en suivant une série d'équivalences qui, elles, varient selon les périodes et les lieux.

Ainsi, quelques siècles plus tard, le nom du roi Pedro $\mathrm{I}^{\mathrm{er}}$, mais cette fois de Castille (1334-1369), apparaît dans une translittération différente sur les inscriptions des alcazars de Séville (fig. 3$)^{37}$.

$C^{\prime}$ est seulement à travers ces exemples que nous pouvons supposer que, dans la Péninsule ibérique médiévale, les chrétiens et les musulmans firent de nombreux efforts pour translittérer dans leurs langues à l' aide des alphabets qu' ils utilisaient les noms propres de la culture de l'Autre. Cela ne signifie cependant pas qu'il n'y ait pas eu une forme homogène pour le faire comme

36 Au lieu de «ráš(a)m Béțo (a)ben Šánğo » [ «rášam Péțro aben Šánčo»], du latin «Signum Petri Sancii», il lit «Rey Pedro aben Sancho». Hernández («En la prehistoria», p. 276) interprète la signature comme un «signo aljamiado del "rey Pedro aben Sancho" », reproduisant la lecture erronée d'Ubieto: «rex Pedro ben Xancho» (Colección diplomática, p. 18).

37 Valencia, «Las inscripciones árabes», p. 295. Je remercie Rafael Valencia pour l' envoi de son article mais aussi des photos et de la lecture correcte des inscriptions. Voir aussi Marquer, Propagande politique, p. 462. Bien que Marquer lise « dion Biḍru», la lecture correcte semble être « dọ Bidru», l' emphatique étant utilisée seulement pour le titre et non pour le nom du roi. 
le montrent les inscriptions qui ont été mentionnées; mais il a pu exister un nombre élevé de possibilités de translittérations. Parmi les Morisques d'Almonacid de la Sierra circulait par exemple une feuille aujourd'hui perdue avec un texte transcrit de forme curieuse ${ }^{38}$. De la même manière, dans un manuscrit en arabe de la fin du XVII ${ }^{\mathrm{e}} \mathrm{s}$. ou début du XVIII ${ }^{\mathrm{e}}$, un système de translittération complètement différent de celui qu' avaient adopté les Mudéjares et les Morisques a été employé (cf. §4). Toutefois, cet exemplaire conservé à Londres contient une bonne partie du texte d'un des manuscrits d' Almonacid ${ }^{39}$, ce qui signifie qu'il partage un même original avec une copie morisque et recourt, malgré cela, à un autre type de translittération (mis au point pour la circonstance ou copié $)^{40}$.

Par contre, j' ai fait allusion au «système de translittération» adopté par les Mudéjares et les Moirisques. Les textes copiés et produits au sein de ces communautés présentent une grande homogénéité en ce qui concerne le système de translittération. Nous pouvons estimer que ce système dut se développer au cours d'une période antérieure à 1462 étant donné que l'œuvre composée à cette date par 'Iça Gidelli, le Breviario sunní ${ }^{41}$, présente déjà un système stable qui a été repris pour toute la production postérieure des Mudéjares et des Morisques, avec un très petit nombre d'élémentrs variables (principalement la façon de rendre les diphtongues et l' emploi de la shadda ${ }^{42}$.

La majeure partie de la production mudéjare et morisque est en caractères arabes; celle qui nous est parvenue a été transcrite pendant la seconde moitié du XVI ${ }^{\mathrm{e}}$ s. Pendant longtemps, les savants ont fait valoir que Mudéjares et Morisques utilisaient l'alphabet arabe afin de dissimuler à l'Inquisition leurs textes écrits, mais cette hypothèse perd de plus en plus de terrain. Bien au contraire, écrire des textes avec ces lettres, même si la langue était en grande partie du castillan, les rendait automatiquement suspects d'être des écrits religieux musulmans, ce qui était complètement interdit et sévèrement puni depuis le début du Xvi ${ }^{\mathrm{e}} \mathrm{s}$. lorsque le cardinal Cisneros fit brûler publiquement des centaines de manuscrits sur la place de Bibarrambla à Grenade afin de dissiper quelque doute que ce soit sur l'interdiction qu' il voulait mettre en place.

Il est vrai que, pour bien des savants depuis le XVII ${ }^{\mathrm{e}} \mathrm{s}$. jusqu'à notre époque, ces textes pouvaient difficilement être déchiffrés: on pensait qu'il s'agissait

38 Ribera et Asín, Manuscritos árabes.

39 Madrid, CCHS-CSIC, TNT, RESC/22. Edité par Labarta, Libro de los dichos.

40 Castilla, «A la búsqueda».

41 Les copies conservées datent cependant du XVI ${ }^{\mathrm{e}}$ s. Voir $§ 3.2 .2$.

42 Castilla, «Uses and written practices». 
de textes «persans $»^{43}$, africains ${ }^{44}$ ou encore de musique. Mais nous savons que, avec ou sans l' aide des Morisques eux-mêmes, les agents de l'Inquisition étaient parfaitement au courant de l'existence de cette production et pouvaient avoir accès aux contenus de ces livres. Nous le savons grâce à un document qui a été conservé et où on peut lire: «Declaraciones hechas en un proceso formado en la Ynquisición de Zaragoza contra Rodrigo el Rubio, vecino de Albeta. Morisco, esto es, moro convertido al cristianismo o descendiente de Moros. Año 1567. En la quales declaraciones consta el contenido del presente libro arábigo $»^{45}$. Le texte rapporte l' histoire d'un homme, possesseur et probablement copiste d'un manuscrit aljamiado, qui avait été dénoncé par un voisin ${ }^{46}$. Dans le document, les contenus du livre, qui étaient des miscellanées comme l'essentiel de cette production, sont décrits avec précision. La déclaration devant le tribunal est exacte puisque nous pouvons le vérifier avec le manuscrit qui a été préservé de même que sa description au $\mathrm{XvI}^{\mathrm{e}} \mathrm{s}$. Un élément supplémentaire se trouve dans la capacité que les traducteurs officiels du royaume avaient de comprendre les textes aljamiados ${ }^{47}$.

Même si cela paraît curieux, dans ce type d'écriture arabe, il n'y a pas seulement de nombreux chapitres qui sont en relation avec la religion et la culture musulmanes, mais également des prières chrétiennes ${ }^{48}$. Les Morisques, forcés de se convertir au christianisme en 1502 en Castille et en 1526 en Aragon, devaient apprendre les rudiments de leur nouvelle religion: le Notre Père, l'Ave Maria, le Credo, le Salve Regina, les Dix commandements et les quatorze articles de la Foi ${ }^{49}$. Pour des communautés qui ne connaissaient pas l' alphabet latin, la seule façon de rendre ces aide-mémoire utiles était d'écrire ces textes en castillan, en latin ou en catalan, selon les nécessités ou les exigences du prêtre local, mais en caractères arabes ${ }^{50}$.

43 Voir par exemple la note de Muscat au début du manuscrit Madrid, BNE 5310: «No le entiendo. Es persiano»; ou celle dans Madrid, BNE Mss/Res. 245: «El Mancebo de Arévalo. Está en letra persiana. Tratado de exercicios espirituales», les mots «Está en letra persiana» ayant été barrés.

44 Selon la notice du catalogue de Cambridge, UL, Or. $65^{2}$ ou Valence, Médiathèque publique et universitaire, ms. 39. Voir Castilla, «A la búsqueda».

45 Paris, BnF Esp. 93. Castilla, Les manuscrits mudéjares et morisques.

46 Paris, BnF Arabe 1163. Edité partiellement par Suárez Piñera, Publicación y estudios.

47 Madrid, BNE MSs/5306, une note sur le premier feuillet écrit a été ajoutée par Andrés de San Juan, traducteur du roi pour les langues orientales qui indique le contenu du livre et la façon de lire les caractères arabes.

48 Labarta, «Oraciones cristianas»; Labarta et Barceló, «Latín y romance».

49 Domínguez Ortiz et Vincent, Historia de los moriscos, p. 96.

$5^{\circ} \quad$ Labarta, «Oraciones cristianas», p. 178, n. 8. 
Dans ces mêmes caractères, on peut également lire des textes de divertissement qui ne viennent pas seulement de la culture arabo-musulmane ${ }^{51}$, mais sont aussi des textes à succès qui circulaient dans l'Europe chrétienne, comme Los amores de París y Viana ${ }^{52}$, ou des compositions nouvelles, à l'instar des guides de voyage pour rejoindre Venise ou Constantinople ${ }^{53}$.

\subsubsection{Caractères latins}

Nous savons par le texte même des prologues de certaines œuvres que l' auteur avait décidé de rédiger son livre en castillan en raison de l'ignorance de l' arabe des ses destinataires; ignorance du lecteur qui peut être déduite à l'occasion par comparaison des passages monolingues ou bilingues de la même tradition textuelle ${ }^{54}$. D'un autre côté, même si c' est exceptionnel, l' auteur peut s' excuser d' avoir utilisé les caractères latins en faisant valoir qu' il était dans l'obligation de le faire à cause de l' ignorance où étaient ses lecteurs potentiels de «la letra de los muslimes»:

Está escrito en letra de cristianos [...] está más a vista de los muslimes que saben leer el cristiano y no la letra de muslimes. Porque es cierto que dixo el annabí Muhammad $\left[s^{\mathrm{c}} \mathrm{m}\right]$ que la mejor lengua era la que se entendía ${ }^{55}$.

Le phénomène est le contraire de ce que nous avons vu dans le point précédent $(\S 1$.3.1) lorsque j' ai fait allusion aux prières chrétiennes écrites par les Morisques de Valence en caractères arabes en raison de leur méconnaissance de l'alphabet latin; ou à l'explication donnée par Cervera pour expliquer un document d' archive rédigé en castillan et signé par deux morisques avec leurs noms chrétiens mais en caractères arabes ${ }^{56}$.

51 Montaner, «La literatura aljamiada».

52 Galmés de Fuentes, Historia de los amores.

53 Lopez-Baralt et Irizarry, «Dos itinerarios».

54 Martínez de Castilla, Una biblioteca morisca, suggérait - grâce à l'étude ecdotique des différents chapitres d'Aix-en-Provence, Bib. Méjanes Ms. 1223 et Madrid, BRAH 11/9415 - que le destinataire du premier possédait une connaissance de l'arabe (et probablement des rites musulmans) supérieure à celle du possesseur du manuscrit de Madrid et que, à partir de là, les mêmes chapitres, bien que relevant de la même tradition textuelle, contenaient moins de passages en arabe dans le cas du manuscrit de l'Académie.

55 Toledo, В Р 2235, ff. 81 $1^{\mathrm{v}-82^{\mathrm{r}}}$, apud Bernabé, «Interferencias», pp. 110-111, où il offre aussi d' autres exemples de copistes morisques que signalaient le manque de connaissances de l' arabe chez le destinataire.

56 Archivo Histórico Provincial de Zaragoza, Miguel de Longares, 1539. Cervera, Manuscritos moriscos, p. 27 . 
Tout comme cela se passe dans la production en caractères arabes, de nombreux textes de contenu musulman sont écrits en caractères latins. Ainsi, des oraisons musulmanes traduites en castillan ${ }^{57}$, des textes qui contiennent des références au Coran et au Prophète, des traductions d' extraits du Coran très fidèles à l'original ${ }^{58}$ ou même de l' ensemble du texte coranique, accompagné de nombreux commentaires, sans le texte arabe de référence ${ }^{59}$. Il existe aussi des copies de quelques sourates du Coran en arabe, mais transcrites en alphabet latin ${ }^{60}$. Dans certains de ces textes religieux musulmans, Allah est écrit en caractères arabes ${ }^{61}$, mais cela reste exceptionnel; dans la majorité des manuscrits en caractères latins, la référence à la divinité se fait dans le même type d'écriture. De même, le mot «Dios» écrit en caractères arabes a été inséré dans quelques manuscrits ${ }^{62}$. Les Mudéjares et les Morisques copiaient en caractères latins des textes grammaticaux dans lesquels apparait l'équivalent phonétique des lettres arabes ${ }^{63}$, mais aussi des romans chrétiens ou des poèmes de Garcilaso de la Vega ou de Lope de Vega ${ }^{64}$. Cet accès à la littérature chrétienne de la part des communautés mudéjares et morisques ne passe pas seulement par la transmission orale et manuscrite, mais aussi par le biais de l'imprimé (seulement en caractères latins), comme c'est le cas des romances et des chants de Noël («villancicos») castillans qui ont été retrouvés dans le fonds d' Almonacid de la Sierra ${ }^{65}$.

\subsubsection{Mélange de caractères arabes et latins}

Pendant longtemps, quoiqu'il n'en ait pas toujours été ainsi - comme cela a été démontré $-{ }^{66}$ on a défendu l'idée que l'utilisation des caractères arabes par les Mudéjares et les Morisques était propre aux textes produits dans la Péninsule ibérique, alors que les Morisques écrivaient en caractères latins quand ils étaient en exil. En fait, comme je vais essayer de le montrer, l'emploi des

57 Madrid, CCHS-CSIC, TNT, Resc/98E. Dans ce cas, «Allah» est en caractères arabes, mais cela est exceptionnel.

58 Paris, BnF Arabe 447. Cf. Martínez de Castilla, «Corán de Salónica», image dans la p. 231.

59 Tolède, Biblioteca Pública, T235. Publié par López-Morillas, El Corán de Toledo.

6o Par ex., dans Madrid, BNE MSs/9067. Malheureusement, d'autres passages du Coran en caractères latins dont nous connaissons l' existence par des catalogues ont disparu.

61 Voir les documents cités dans les n. 57 et 58 (Resc/98E y BnF Arabe 447).

$62 \quad$ Historia de los amores de París y Viana (cf. éd. Galmés de Fuentes).

63 Madrid, CCHS-CSIC, TNT, Resc/98.3.

64 Madrid, вRAн 11/9394 (olim s-2) (éd. Galmés de Fuentes, Tratado de los dos caminos). Galmés de Fuentes, Los manuscritos, p. 20.

65 Feuilles provenant des ais du manuscrit Madrid, cCHs-CSIC, TNT, RESC/93. Ribera et Asín (éds.), Manuscritos árabes y aljamiados, p. 249.

66 Voir, par exemple, Suárez García, El compendio à partir d'un manuscrit en écriture latine. 
caractères arabes ou latins ne semble pas être systématiquement associé à un type de contenu avec l'objectif de «maintenir leur littérature secrète, hors de l'atteinte de la société environnante, aussi bien en terre chrétienne que dans le monde musulman ${ }^{67}$; il semble davantage lié au type d'écriture avec lequel le copiste, l' auteur ou le lecteur se sentait plus à l' aise et qu' il contrôlait le mieux. Nous pouvons donc trouver des textes de différents types pour la copie desquels l'alphabet latin ou arabe a été employé. Dans certains cas, ce choix paraît même aléatoire comme cela arrive avec ces textes qui relèvent de la même tradition textuelle mais qui, en fonction du manuscrit (et pour cela il faut tenir compte de tous les éléments qui font partie de la fonction de communication), étaient copiés dans l'un ou l' autre type d'écriture.

Ainsi, nous trouvons dans deux copies une oraison de même tradition textuelle qui est récitée pour célébrer les «fadas de la criatura ${ }^{68}$. Au cours de cette fête, le nouveau-né est accueilli dans la communauté musulmane et, à cette occasion, on lui donne un nom. Le premier des deux manuscrits est copié en caractères arabes alors que le second emploie l' alphabet latin. Cela se reproduit pour d'autres copies de cette même prière, en fonction du manuscrit. En voici un extrait:

Señor Allah, aprovecha con este nacido a los leedores y a los escuchadores de tu Alcorán el onrado; y mételo que sea este nacido de su conpañía d' ellos; y mételos en el alfadila y gualardón aparçonantes, y de los mandamientos de tu Alquiteb el onrado obrantes. Y no ay fuerça ni poder sino con Allah el Alto, Grande.

Después tomará en braços la criatura su padre y, en su ausencia, quien más parentesco tendrá con ella, y dirás: $[. . .]^{69}$

Ce fragment, emprunté au manuscrit de Madrid s'écarte légèrement du paragraphe de Tolède (вР, 232) en deux endroits seulement: au lieu de «mételo», la copie tolédane donne «mete»; et au lieu de «d'ellos», «d'ello» - et ce par erreur. Il faudrait donc avancer des hypothèses sur ce changement de graphie non pas en partant du contenu, mais de l'émetteur et du récepteur, c' est-à-dire en fonction des compétences du copiste et des nécessités du lecteur.

En plus du cas que je viens d'exposer (même texte écrit dans deux alphabets différents dans ces deux manuscrits), il existe aussi des exemples de combinaison d'alphabets dans le même volume. Bien que, dans la majorité des

67 García-Arenal, «Ríos y caminos», consulté le 15 décembre 2016.

68 Madrid, BRAH, 11/9415 et Tolède, BP, 232.

69 Madrid, BRAH 11/9415, edité par Martínez de Castilla, El manuscrito aljamiado T19, p. 564. 


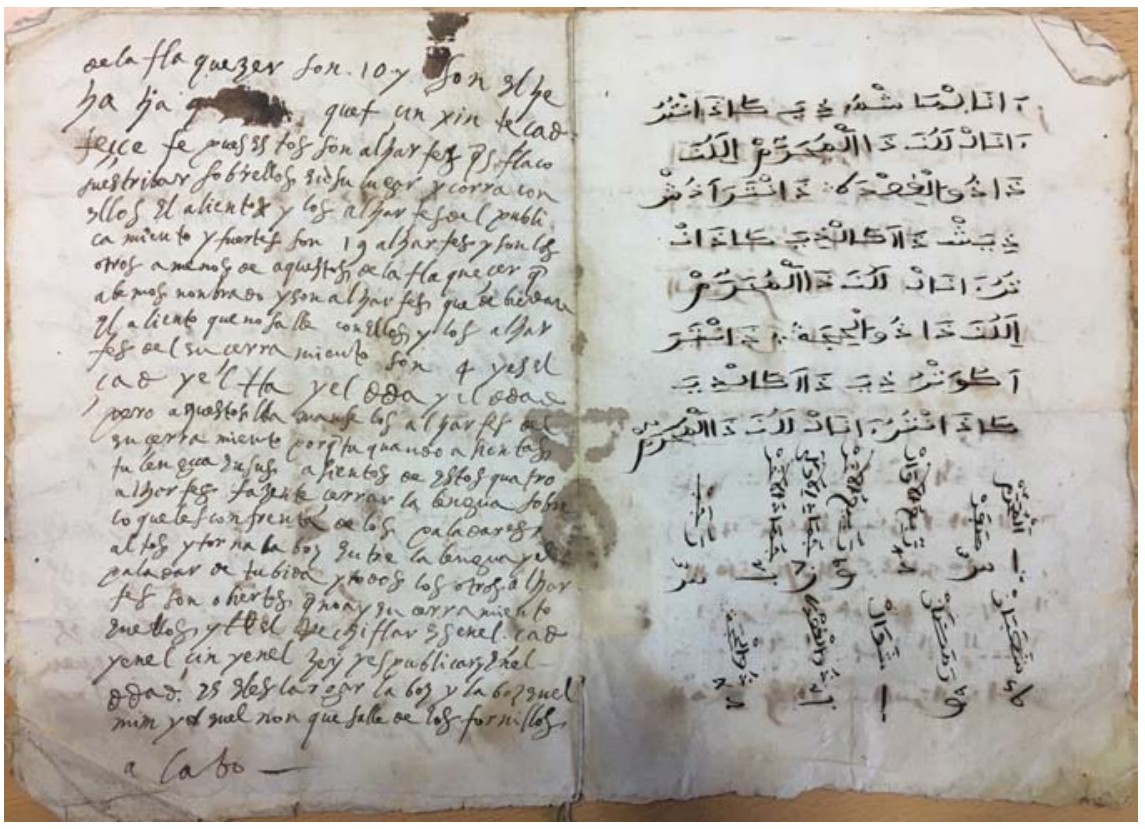

FIGURE 4 Madrid, CCHS-CSIC, TNT, RESC/98.3, ff. $2^{\mathrm{v}}-3^{\mathrm{r}}$ (bifeuillet interne)

(C)CSIC, CCHS, BIBLIOTECA TNT

manuscrits, qu'ils soient unitaires, miscellanées ou factices, on ne trouve qu' un seul type d'écriture, dans les documents et les feuilles volantes incorporés aux reliures des manuscrits trouvés à Almonacid de la Sierra ou transformés en feuillets de garde, de nombreux textes combinent graphie arabe et latine ainsi que les langues, arabe et espagnol. Dans quelques cas exceptionnels, comme le traité médical conservé à la BRAH, deux types d'écriture et trois langues (castillan dans les deux variantes présentées au §1.2, arabe et latin) sont utilisés tout au long de cette copie, parfois sur le même feuillet (voir ci-dessus).

Dans l'ensemble de ce répertoire de textes, les lettres latines peuvent arriver parfois à être très soignées et à présenter des situations variées: nous trouvons des documents dans une écriture courtisane ou humaniste, des écrits qui ne sont pas toujours composés ou copiés par des Mudéjares ou des Morisques, mais dans l'Espagne médiévale chrétienne, et pourtant utilisés par eux dans un but ou dans un autre (fig. 1).

Dans d'autres cas, il s'agit de textes copiés par les Morisques eux-mêmes. Dans un même binion par exemple, cousu dans ses parties supérieure et inférieure, et formant probablement une seule unité codicologique, figurent deux chapitres qui offrent un texte morisque écrit en caractères arabes et un autre en caractères latins (fig. 4). Le premier est en lien avec le calendrier musulman, le 


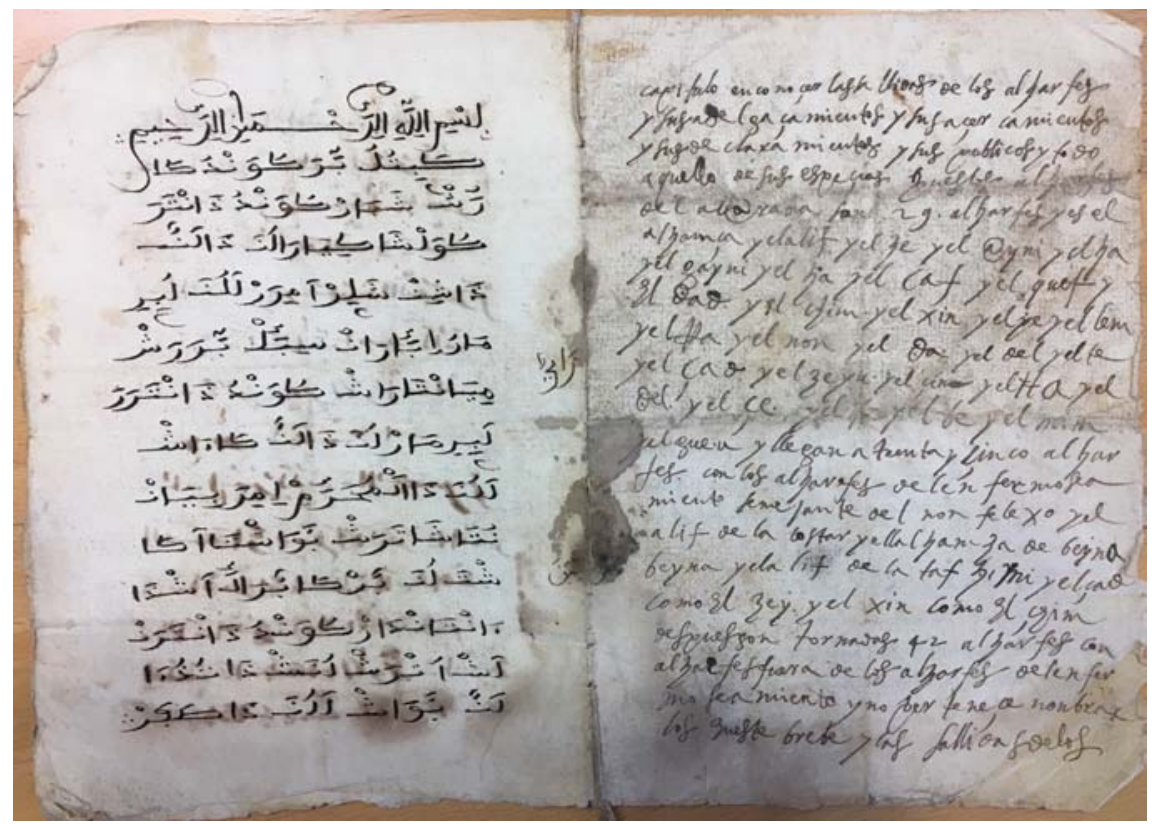

FIGURE 5 Madrid, CCHS-CSIC, TNT, RESC/98.3, ff. $1^{\mathrm{r}} / 4^{\mathrm{v}}$ (bifeuillet externe)

(C)CSIC, CCHS, BIBLIOTECA TNT

second avec la façon de prononcer les lettres de l' arabe. Si le lecteur commence sa lecture par un texte ou par l'autre, il se voit obligé de sauter à l' autre extrémité du binion pour pouvoir lire l' autre chapitre, écrit lui aussi en castillan, mais dans une autre graphie dont la lecture se déroule dans le sens inverse de l'autre. Il existe une seule exception; au f. $\mathrm{r}^{\mathrm{r}}$ on peut lire deux mots en caractères arabes dans la marge intérieure: «zay» et «bayna» (fig. 5$)^{70}$. Ecrites par une main différente et avec une autre encre, elles ne paraissent avoir aucune relation avec le texte écrit en caractères arabes sur ce même feuillet. Elles ont pourtant leur raison d'être. Elles font en fait référence au texte en caractères latins copiés au f. $4^{\mathrm{v}}$. Il s' agit de deux mots que, probablement, le copiste du texte en caractères latins lui-même ou un de ses lecteurs a dû expliquer parce que la forme employée dans le corps du texte ne se comprenait pas suffisamment bien. Ce qui retient l'attention, c' est que celui qui a ajouté ces deux mots, apparemment avec la même encre que celle employée pour le texte en caractères latins, l' ait fait dans la marge de l' autre feuillet alors qu' il aurait pu inclure

70 J'ai tenu en compte la séquence de lecture de l' arabe, de droite à gauche, mais le contraire aurait également été possible. 
ces explications dans un endroit plus proche, par exemple l'interligne ou la marge intérieure du f. $4^{v}$ elle-même. Comme il s' agit du feuillet externe du binion (fig. 5), le lecteur moderne peut ne pas saisir la fonction de ces deux mots s'il ne procède pas à une lecture attentive. Peut-être que cela était l' objectif et l'explication de l'inclusion de ces mots à cet endroit: ils pouvaient servir d' aide à la lecture ou à la prononciation, mais sans trop attirer l'attention.

L' usage et la connaissance des deux graphies par les Morisques se manifeste dans les exercices de calligraphie qui figurent dans leurs papiers, exercices réalisés tant en caractères arabes ${ }^{71}$ que latins ${ }^{72}$.

Comme je le signalais au début du point I.3, cette utilisation de langues et de caractères se retrouve non pas seulement dans des restes de manuscrits entiers, perdus aujourd' hui, et dans des documents périmés, ce qui en faisait des matériaux de choix pour être transformés en carton, mais aussi sur les feuillets de garde de copies soignées et de grand format ${ }^{73}$. Ainsi, sur la même double page au début d'un volume, deux paragraphes dans deux langues et écritures différentes peuvent apparaître côte-à-côte (fig. 6).

Ce mélange de langues et de graphies se rencontre également quoique de manière exceptionnelle dans des manuscrits entiers. C' est le cas de Madrid, BRAH, 11/9411, un livre de recettes écrit en aljamia en caractères arabes, castillan standard, en arabe et en latin ${ }^{74}$. Au fil des 30 feuillets, on rencontre des textes dans ces trois langues, écrits dans les deux alphabets avec la même encre, ce qui conduit à penser qu'ils ont été transcrits par la même main. Sans mise en page particulière, ni présentation des paragraphes en faisant attention à l'une ou l' autre des caractéristiques linguistique ou graphique, les textes se suivent sur les mêmes pages. Le copiste copiait-il à partir d'un modèle similaire et respectait-il langues et graphies? Ou copiait-il au contraire de différents originaux et suivait-il par commodité les systèmes linguistiques de ces derniers? A quel public s' adressait-il? Comme il s' agissait de recettes, l' ouvrage était-il destiné à un médecin ou à quelqu'un qui connaissait non seulement les langues, mais aussi certaines graphies aussi difficiles à comprendre que le sont celles qui sont conservées dans ce manuscrit?

Quoi qu'il en soit, il semble avoir été à l' aise avec les caractères latins, tant en latin qu' en espagnol, même s'il semblait connaître parfaitement l' arabe langue et alphabet. Et pourtant, les passages en aljamia sont ceux qui trahissent le plus d' incertitude. Le copiste, qui les a toujours vocalisés, a employé la même

\footnotetext{
71 Madrid, CCHS-CSIC, TNT, Resc/101B.6.

72 Madrid, cCHS-CSIC, TNT, Resc/98.4.G.1.

73 Madrid, cCHS-CSIC, TNT, Resc/4.

74 Galmés de Fuentes, Los manuscritos.
} 


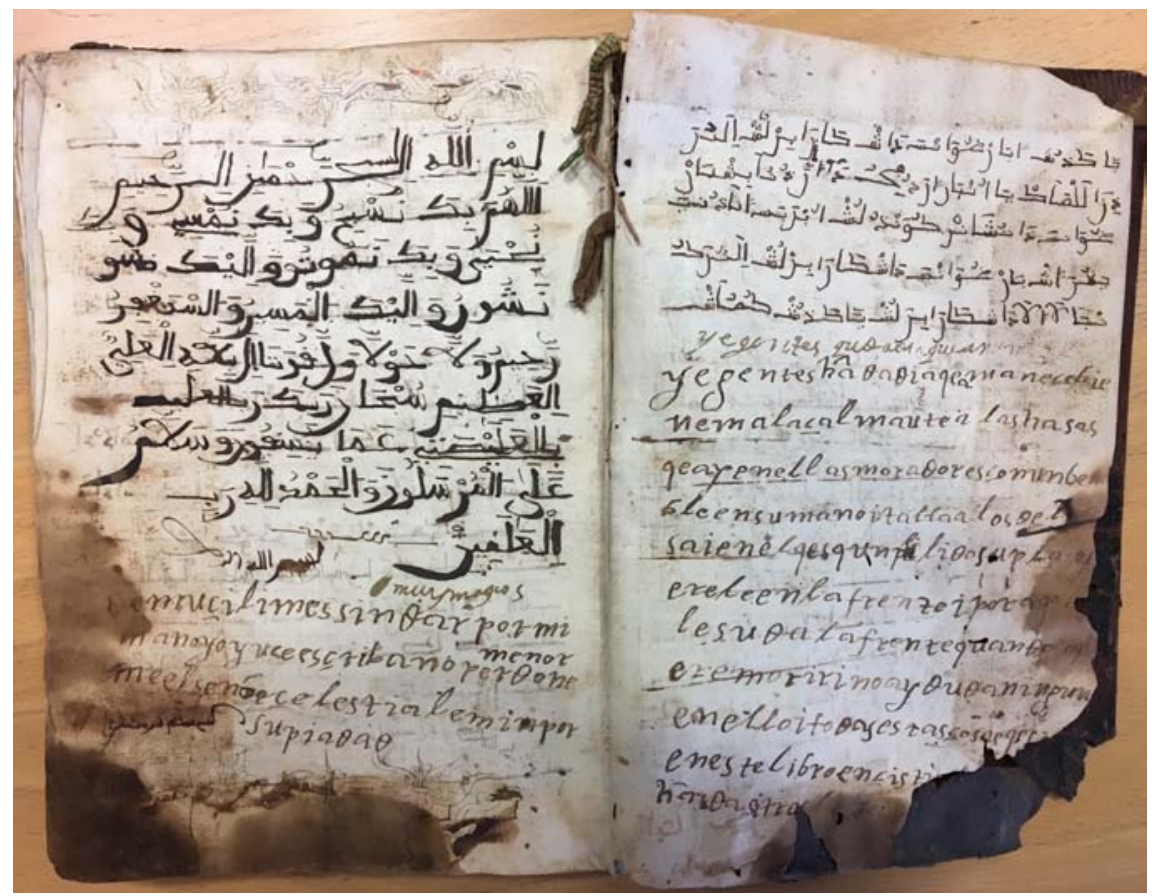

FIGURE 6 Madrid, CCHS-CSIC, TNT, Resc/4, ff. I $\mathrm{I}^{\mathrm{v}} \mathrm{II}^{\mathrm{r}}$ (C)CSIC, CCHS, BIBLIOTECA TNT

encre, le même calame et la même mise en page que pour les autres langues et graphies; mais dans le cas des paragraphes en aljamia, l'écriture est moins cursive, plus anguleuse et tassée que celle où il s' agit d' arabe. De manière générale, le copiste paraît se sentir moins à l' aise avec ce type d'écriture et de langue que pour le reste; en fait, il semble avoir intégré de manière spontanée au texte aljamiado une série de commentaires, non pas en aljamia, mais en arabe. De même, il a inclus des traductions interlinéaires en arabe pour quelques mots latins.

F. $6^{\mathrm{ro}}$. En caractères arabes. Arabe:

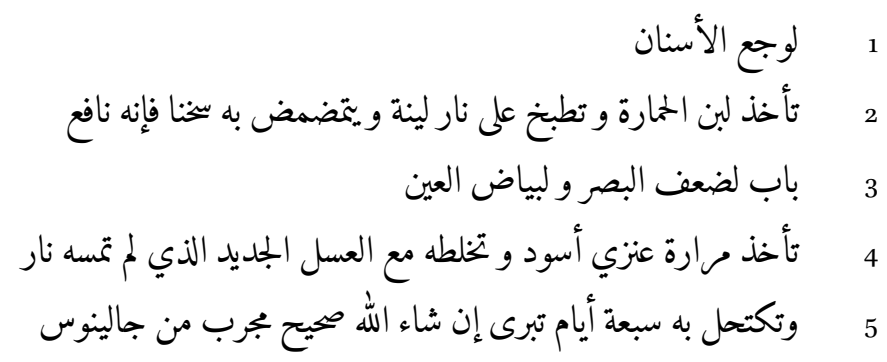


En caractères arabes. Aljamia et arabe:

6 Para la fiebre $[\dot{i}$ ?] mores. Sea tomada una calabaça y d[e]xada en el forno.

7 Y endepués agujerarla. Y aquella agua que saldrá d'ella, replegarla. Tome

8 de la media escudilla una mañana sin otra cura más بالعرض

$9 \quad$ Mas 155

De la même manière, à la fin de la feuille, aljamia et arabe sont mêlés dans une même phrase:

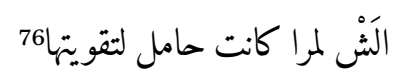

F. 6v . En caractères latins. Castillan:

8 Sea tomado una corteza de raíz de nog[u]era verde cortada

9 debaxo de tierra antes que salga el sol y sea redodiada

1o como vna miaja y metida donde quede hazer la fue

11 nte y a toda allí dentro poco espacio hará llaga. Sea

12 regido como se acostumbra regir y es que después de

13 fecha les $[. .$.$] le ponga ma[n]teca para aloarla$

14 y después ponelle fuera un poquito para tenerla abie

15 rta y así cada el día sea limpiada. Y purgará por allí

16 todos estos malos humores que allí hubiere. Dize

17 que lo pratican los vizcaýnos mucho y con aquello

$18 \quad$ se cauteriza ${ }^{77}$

Les trois langues (arabe, latin et espagnol standard/aljamia ou espagnol arabisé en caractères arabes) et les deux alphabets (arabe et latin) coexistent sur le même feuillet, écrit probablement par le même copiste et destiné a priori à un destinataire capable de les comprendre tous ${ }^{78}$.

75 «J' ai entendu dire que ce remède soulagerait les maux d' estomac, l' indigestion et les gaz».

76 [Aljamia:] «A las» [Arabe:] «Pour la femme enceinte, pour avoir de l'énergie».

77 Je remercie chaleureusement Fernando del Ser pour sa proposition de lecture de ce passage.

78 L'écriture du texte latin est très difficile à déchiffrer. Je remercie très vivement Roland Béhar pour son aide pour lire le passage en latin de ce feuillet. Cependant, la lecture est encore incomplète, et c' est la raison pour laquelle je ne la publie pas ici. 


\subsubsection{Caractère hébreux}

Parmi les graphies et les langues rencontrées dans ces collections, figure aussi l'aljamia aragonaise hébraïque, c' est-à-dire l'aragonais en caractères hébreux. L' exemple le plus significatif a été découvert dans les ais de la reliure du manuscrit Madrid, CCHS-CSIC, TNT, Resc $/ 34^{79}$. Il s'agit d'une copie de la troisième partie du Coran, conservée actuellement à l'état de fragment sans ordre ${ }^{80}$. Dans les ais de cette reliure apparut également, parmi d'autres choses, un registre comptable de paiements de l'aljama de Tarazona réalisés entre 1406 et 1407, et a été catalogué précisément par Ribera et Asín ${ }^{81}$. Il a disparu des fonds de la collection d'Almonacid, conservée dans un premier temps à la Junta para Ampliacion de Estudios, ensuite par le csic, et se trouve de nos jours à la Jewish

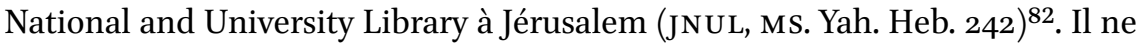
reste dans la bibliothèque du csic que de petits fragments indéchiffrables, provenant probablement de ce même document.

On considère habituellement comme aljamia le castillan écrit en caractères arabes employé par les Mudéjares et les Morisques, c'est-à-dire les dernières communautés musulmanes qui vécurent dans la Péninsule ibérique avant l'expulsion définitive des Morisques en 16o9-1614.

Si nous revenons à la seconde définition d' «aljamia» par la Real Academia Española, nous voyons qu'il s'agit d' «un texte morisque en langue romane, mais transcrit en caractères arabes ». Comme nous avons déjà abordé la question de l'écriture, nous nous tournerons maintenant vers le problème que pose l'introduction d' un nouveau concept, celui de «texte morisque», étant donné que la définition déborde le champ de la linguistique pour entrer dans celui de la culture et de la religion.

On peut dire qu' un texte morisque (à quoi il faudrait ajouter mudéjar, pour des raisons chronologiques) est:

a. Un texte produit, traduit ou copié par un Mudéjar/Morisque et destiné à la communauté mudéjare/morisque (majorité des cas).

b. Un texte créé ou copié dans cette communauté, mais destiné à une utilisation extérieure (ex., le Llibre de çunna y xara).

79 Madrid, CCHS-CSIC, TNT, Resc/98.1.

8o Martínez de Castilla, «Qur’anic Manuscripts», p. 108.

81 Ribera et Asín, Manuscritos árabes, p. 133 et 251.

82 Castaño, «Nuevos documentos» et « Kettubot en-cubiertas». 
c. Un texte créé en dehors de la communauté morisque, mais transcrit par des Morisque pour une utilisation interne (ex., les Amores de París y Viana).

d. Un texte créé et copié en dehors des communautés mudéjares/ morisques, destiné à être lu au début pas des chrétiens ou des juifs, mais dont les copies circulaient dans les communautés mudéjares/ morisques (ex. des imprimés, textes en aljamia hébraïque).

Bien que la majorité des textes conservés appartiennent au groupe a) et que d' autres peuvent aisément prendre place dans les autres catégories que je propose, il n' est pas toujours facile de ranger de manière claire les différents documents et manuscrits mudéjares et morisques qui ont été préservés dans l'un ou l'autre groupe, particulièrement ces textes en langue romane qui ne sont pas caractérisés linguistiquement par l' arabisme, l' archaïsme ou l' aragonisme ou encore ceux qui ne sont pas translittérés en cacatères arabes, ce qui pourrait être le cas de Paris, BnF, Arabe $447^{83}$. Il s' agit d'une traduction interlinéaire d' une série d' extraits du Coran en castillan standard, sans aucun autre lien avec celles qui circulaient parmi les Morisques que la sélection de versets ${ }^{84}$, cette copie ayant été achevée à Salonique en 1568 en une élégante écriture humaniste par un certain Ibrahim Izquierdo. Il faudrait disposer de davantage de données à propos du copiste, du destinataire et de l'usage réservé à cette copie à Salonique dans la seconde moitié $\mathrm{du} \mathrm{XVI}^{\mathrm{e}} \mathrm{s}$. pour pouvoir être sûr de classer correctement ce manuscrit dans une des catégories de la typologie que j' ai proposée (cf. §4).

Pour Qui (Destinataire)

\subsection{Les communautés mudéjares et morisques elles-mêmes}

La majorité des textes traduits et copiés par des Mudéjares ou des Morisques ont été transcrits pour l' utilisation en interne de ces communautés ${ }^{85}$.

\subsection{Les chrétiens}

Malgré le paradoxe apparent, les destinataires de quelques-unes de ces copies produites au sein des communautés mudéjares et morisques furent dans quelques cas des chrétiens. Les objectifs paraissent clairs: des affaires religieuses et pratiques. Ainsi, d'un côté, les autorités ecclésiastiques et inquisitoriales esti-

83 Castilla, Manuscrits mudéjares et morisques.

84 López-Morillas, «The Genealogy»; Martínez de Castilla, «Qur’anic Manuscripts ».

85 Wiegers, «Datation», p. 25 . 
maient important de connaître l'islam à des fins de polémique, même si je n' écarte pas qu'il y ait eu un intérêt pour le sujet lui-même dans certains cas. D'un autre côté, afin que la bonne entente règne entre voisins, il était nécessaire de connaître le droit musulman selon lequel chrétiens et musulmans devaient gouverner conjointement à l'époque mudéjare.

\subsubsection{Llibre de la çunna e xara, fin du Xve $\mathrm{s}$.}

De fait, les textes les plus utilisés par les chrétiens ou copiés par des Mudéjares ou des Morisques étaient des traités de droit malikite. D' une part, le Llibre de la çunna exara date de la fin du Xv $\mathrm{Xv}^{\mathrm{e}}{ }^{86}$. Ce fut apparemment « un cadi ou lugarteniente de cadi real» de la région de Játiva ou d'Alzira qui a traduit ce traité de l'arabe au catalan, en lui ajoutant «unas ordenanzas cristianas referidas a los musulmanes ${ }^{87}$. Le destinataire était peut-être le seigneur de Sumarcarcer, à qui avait été concédée la suzeraineté sur cet endroit en 1464, et qui, selon les fueros (codes) de Valence, devait rendre justice, conseillé par un cadi musul$\operatorname{man}^{88}$.

\subsubsection{Breviario sunní, 1462}

D'un autre côté figurent les copies d' un autre traité juridique musulman composéà la même époque:le Breviario sunní. Bien que la différence de perspective soit claire, étant donné que le Breviario était destiné dans un premier temps aux musulmans et non aux chrétiens (ce qui était en revanche le cas du traité valencien), il semble qu'il circula en caractères arabes et latins dès le début et qu'il fut utilisé par des musulmans et des chrétiens. Il fut rédigé par le faqīh de Ségovie Iça Gidelli en 1462 et toutes les copies conservées sont plus récentes. Parmi celles qui ont été perdues (ou dont le lieu de conservation est inconnu), il s' en trouve deux auxquelles fait référence l' inventaire de la bibliothèque de Leonor de Pimentel ${ }^{89}$ : «un libro de coberturas de cuero morado de don Yça Guidili alfaquí de los moros de Segovia, que hiso contra la fe, al cual responde fray Juan Lopes» $\mathrm{y}$ un «Libro de coberturas moradas escripto de mano qu' es el que hiso don Caguidili, moftí de los moros de Segovia ${ }^{90}$. La première description semble se rapporter à l'ouvrage, aujourd'hui perdu, composé par le théologien Juan Lopez de Salamanque pour réfuter ce traité rédigé, selon l' inventaire, «contre la foi». Nous savons de fait que le livre de 'Iça Gidelli était suffisam-

86 Voir n. 11.

87 Selon l'hypothèse de Barceló, Un tratado catalán, p. XIV.

88 Ibidem.

89 Leonor de Pimentel était une comtesse castillane qui vécut dans la seconde moitié du $\mathrm{xv}^{\mathrm{e}} \mathrm{s}$.

9o Wiegers, «Breviario Çunní». 
ment important et connu, même dans des milieux chrétiens, pour susciter une réfutation seulement quelques années plus tard. Il semble logique de penser que les chrétiens lurent son ouvrage en caractères latins pour des raisons pratiques parce qu'il était ainsi plus facile à déchiffrer qu' une copies en alphabet arabe. Cependant, la diffusion supposée du texte en caractères latins à une date précoce (1462), comme le soutient Wiegers ${ }^{91}$, soulève à son tour une série de questions. Comment et quand le texte fut-il transcrit en alphabet latin? Qui s'en chargea? Cela fut-il fait pour un commanditaire morisque ou chrétien? Une bonne partie des livres de la comtesse furent transcrits, achetés, hérités ou reçus en présent: mais, comme l' avait déjà remarqué Jiménez Moreno, «lo más probable es que los condes dispusieran de algún escribano al servicio de la familia encargado de copiar ciertos libros ${ }^{92}$; pareillement, «no se puede descartar tampoco que la condesa o su hijo Juan de Zúñiga contaran con algún iluminador personal, que podría ser el mismo que decoró la inicial de una esmerada copia del testamento de doña Leonor ${ }^{93}$. Pouvons-nous supposer que ce copiste fut aussi chargé de la transcription du Breviario sunní? Wiegers pense que 'Iça et d'autres Mudéjares furent probablement responsables de ces copies ${ }^{94}$. D'un autre côté, si le traité était si dangereux qu'une réponse chrétienne presque immédiate dut lui être faite, pourquoi deux copies avec une reliure violette et copiées par 'Iça Gidelli faisaient-elles partie de la bibliothèque aux contenus très pieux qui appartenait à une noble dame de la seconde moitié du XVe siècle? Peut-être faut-il envisager l'hypothèse qu'il existait des lectures différentes à celle qui peut être faite dans une perspective strictement musulmane, bien loin de la polémique religieuse, et que nous examinerons à la suite.

Plusieurs exemplaires du Breviario sunní furent copiés par l'Inquisition pour son propre usage: le livre qui, en principe, avait été rédigé pour rassembler, de manière brève et simple, les fondements des pratiques religieuses que tout bon musulman devait suivre se convertit en un ouvrage fondamental pour la lutte de l'Inquisition contre ces communautés. Représentatifs de cette destinée sont des copies comme celle qui se trouve dans l'Archivo General de la Nación à Mexique $^{95}$ ou une autre à Madrid ${ }^{96}$.

\footnotetext{
91 Wiegers, Islamic Literature, pp. 137-139.

92 AHN. Sección Nobleza. OsUNA. C.217.D.21. Apud Jiménez Moreno, «Formación, uso y dispersión», p. 658 .

93 Ibidem, p. 658, n. 13.

94 Wiegers, Islamic Literature, p. 140.

95 México, Archivo General de la Nación, Ramo Inquisición, núm. 1528 (54): 1. Feliciano, «Breviario çunní».

96 Madrid, BNE MSS/2O76.
} 
On ne sait pas avec précision à quelle date des documents et des manuscrits complets qui n'étaient pas en arabe ont commencé être produit au sein des communautés mudéjares et morisques. Les dates avancées vont de la fin du $\mathrm{XIV}^{\mathrm{e}} \mathrm{s}$. au début du XVII ${ }^{\mathrm{e}}$. La première est appuyée parl' utilisation de la strophe «cuaderna vía» dans le Poème de Yusuf; bien que les copies de cette œuvre soient du XVI ${ }^{\mathrm{e}} \mathrm{s}$., ce type de strophe semble être sortie d' usage à la fin du XIV ${ }^{\mathrm{e} 97}$. D'un autre côté, la date limite pour cette production est fixée par l' expulsion définitive des Morisques en 16o9-1614.

L' emploi du castillan (soit standard, soit dans sa version arabisée) en caractères arabes semble être spécifique des royaumes d'Aragon et de Castille, alors que ceux de Valence et de Grenade continuaient à utiliser l' arabe de manière exclusive. Il existe cependant des exceptions. Nous savons par des documents de l' Inquisition que des papiers en latin, en catalan et en castillan en caractères arabes furent confisqués à des Morisques valenciens ${ }^{98}$ et le Llibre de çunna y xara fut écrit dans le royaume de Valence en catalan en caractères latins. (cf. $\S 1.1$ et I.3). L'Afrique du Nord et les quelques cités ottomanes où se réfugièrent les Morisques exilés d'Espagne furent aussi des lieux de copie en aljamia à la fin du XVI ${ }^{\mathrm{e}}$ s. et au début du XVII ${ }^{\mathrm{e}}$, mais les contenus semblent en grande partie liés à une polémique anti-chrétienne ${ }^{99}$.

Comment définir dans ces conditions les textes en langue romane en caractères arabes retrouvés en dehors de ces limites spatio-temporelles? Quatre cas de manuscrits sont tout à fait significatifs: trois en caractères arabes, un en latin. Des trois premiers, un date du $\mathrm{XxI}^{\mathrm{e}} \mathrm{s}$. (Madrid-Espagne); un autre de la fin du XIX ou début du XXe (Espagne, probablement Madrid); et le dernier de la fin du XVII ${ }^{\mathrm{e}} \mathrm{s}$. ou du début du XVIII $I^{\mathrm{e}}$. Quant au manuscrit en caractères latins, il s' agit d' une copie du XIX ${ }^{\mathrm{e}} \mathrm{s}$. La raison d' être de ces copies est évidemment bien éloignée de l' objectif d' enseignement de l' islam à des communautés mudéjares et morisques, et les copistes de même que les destinataires de ces manuscrits n' appartenaient pas à ces communautés musulmanes qui vivaient dans les royaumes péninsulaires aux $\mathrm{XV}^{\mathrm{e}}$ et $\mathrm{XVI}^{\mathrm{e}} \mathrm{s}$.; aussi faut-il d'abord nous demander si nous avons devant nous des textes morisques ou aljamiados.

97 Menéndez Pidal, Poema de Yúçuf.

98 Labarta, «Oraciones cristianas »; Labarta et Barceló, «Latín y romance».

$99 \quad$ Epalza, Los moriscos antes y después. 


\subsection{Note personnelle. Madrid, 2015}

Le premier texte se trouvait sur la porte d'une maison à Madrid en $2015^{100}$. Cette porte avait apparemment été recyclée, mais la signature qui s'y trouve correspondait au nom d' un jeune voisin d' origine tunisienne. Le texte peut être lu de la façon suivante:

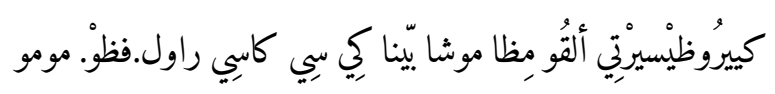

Kyyrū ḍīsīrtī alqū mī ḍā mūšā bbīnā kī sī kāsī Rāūl. Fḍū. Mūmū Quiero decirte algo. Me da mucha pena que se case Raúl. Fdo. Momo

Selon la définition proposée par la Real Academia Española, nous n'avons pas ici un texte aljamiado puisque l' auteur n'est pas morisque (c' est-à-dire qu'il n'a pas été écrit par un more converti ou «Nouveau chrétien»). Cependant, il remplit le reste des conditions: c'est un texte en langue romane écrit en caractères arabes qui suit un type déterminé de conventions dans la translittération. Si nous ne savions pas que le nom du voisin est le même que celui de l'auteur du texte, toute personne de la seconde moitié du $\mathrm{xx}^{\mathrm{e}} \mathrm{s}$. aurait pu l'avoir écrit a condition d' avoir quelques connaissances en espagnol et en écriture arabe. Selon le témoignage de cette inscription, le seul lien avec la culture arabo-islamique est l'écriture elle-même étant donné que le reste (la langue et la disposition du texte) appartient à une culture spécifique, celle de l' Espagne du dernier quart du $\mathrm{Xx}^{\mathrm{e}}$ s. Nous y rencontrons par exemple l' abréviation «fdo.» pour «firmado» («signé») qui a été en usage en Espagne au cours des derniers vingt-cing ans.

Pour l'époque morisque, nous possédons une autre note ou lettre qui, bien que de contenu différent, est tout à fait proche de l'exemple précédent.

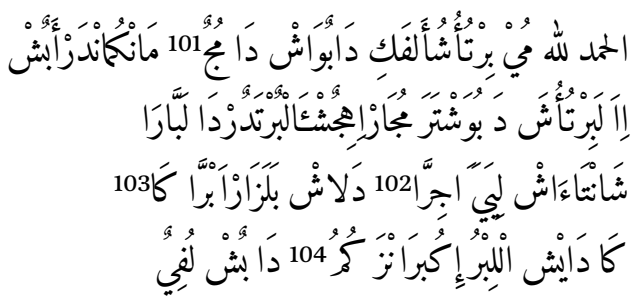

\footnotetext{
100 Je remercie Blanca Villuendas pour l'information sur cette note et pour sa reproduction.

101 Curieusement, le copiste achève le jïm final par un trait qui pourrait être compris comme un $r \bar{a}$, ce qui rend le mot incompréhensible.

102 Voir n. 101, le jïm figurant dans ce cas au milieu d' un mot.

103 Lecture non identifiée.

104 Le trait final du mimm ressemble à celui du jim. Voir n. 101.
} 


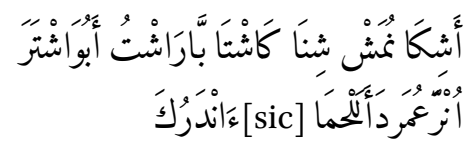

Alḥamdulillah. Muy virtuoso alfaquí: Después de mucho m’encomendar a vos

i a la virtuosa de vuestra mujer i hi[j]os, el portador de la pre sente es Liyaya A[gu]irre; dales plazer [...]

que deis el libro y cobranza como de vos lo fío.

Así que no más sino qu' esté presto a vuestra

onra. 'Umar de la aljama en Daroca ${ }^{105}$

Il s' agit d'une lettre sur papier, apparemment du Xvi ${ }^{\mathrm{e}}$ s., qui avait été envoyée à l' alfaqui de Daroca en Aragon par un de ses concitoyens, 'Umar. L' expéditeur signe de son nom musulman et adresse son texte à l'autorité musulmane de sa petite bourgade, principalement habitée par des Morisques ou «Nouveaux chrétiens ». Le porteur utilise son nom chrétien («Aguirre») et un prénom qui pourrait être musulman, «Liyaya». Si nous examinons la langue, nous pourrions conclure que la lettre a été écrite par une personne cultivée de l'époque, indépendamment de sa religion, même si une touche musulmane est présente avec la hamdala du commencement, le nom de l'auteur de la lettre, celui du porteur, Liyaya, et la profession du destinataire, un alfaqui. Pouvons-nous affirmer que cet exemple est écrit en aljamia, ce qui ne serait pas le cas du précédent, simplement en raison de l' appartenance religieuse de l' auteur ou de la profession du destinataire? Ou encore à cause de la date à laquelle la lettre a été écrite ou transcrite?

Dans le premier exemple, il ne semble pas y avoir un problème de connaissance de l'alphabet latin, bien au contraire: il semble que «Momo» voulait envoyer un message «codé» ou «secret» à quelqu'un. Les possibilités que de nombreuses personnes à Madrid vers la fin du $\mathrm{xx}^{\mathrm{e}} \mathrm{s}$. aient pu comprendre ce système de transcription sont très basses vu qu'il n'y a pas d'habitude de codage de ce type en Espagne (§1.3).

105 Madrid, BNE MSS/5452. Fernández y González a déjà édité ce texte dans Estado socialy político, p. 442: «El hamdolillah (alabanza á Dios) muy virtuoso alfaquí, después de me encomendar á vos é la virtuosa de vuestra muger é hijos, el portador de la presente es Eliah, hijo de Nules, valencian, que le deis el libro é cobranza como de vos fío ». Toutefois, comme on peut le noter, ma lecture est très différente. 


\subsection{Breviario sunní, $X I X^{e} s$. $(\$ 3 \cdot 2.2)$}

Nous avons vu les usages de manuscrits produits ou copiés par des Mudéjares ou des Morisques $(\S 3)$ de la part de chrétiens qui avaient deux intérêts évidents: la polémique religieuse et le droit malikite. Il est plus difficile d'expliquer la présence du Breviario sunní dans une copie en caractères latins du $\mathrm{XIX}^{\mathrm{e}}$ s., ou encore le titre qui apparaît sur le dos de la reliure: «Literatura hebrea $\mathrm{I} »^{106}$.

\subsection{Récits morisques. Madrid (?), fin du $X I X^{e}$ s./début du $X X^{e}$}

Dans la même ligne que le Breviario sunní copié au XIX ${ }^{\mathrm{e}}$ s., dans un des dossiers qui se trouvent dans une boîte de la Bibliothèque nationale d'Espagne, on trouve sept bifeuillets qui réunissent une série de récits en aljamia ${ }^{107}$. Sans date, acéphale et apode, ce manuscrit non relié a été copié à une époque très tardive, probablement la fin du XIX ${ }^{\mathrm{e}}$ s. ou le début du XX ${ }^{\mathrm{e} 108}$. La date de la copie est fournie par le support: il s'agit d'un papier industriel, appelé «de hilo o barba» (papier déchiré aux ficelles) en raison des irrégularités que présentent ses côtés. Cette caractéristique, associée au filigrane «A Serra $S$ », s' explique comme le résultat d'une imitation du papier fait à la main. Ce papier de haute qualité est très connu en Espagne et était principalement utilisé pour faire du papier timbré109. Le nom du fabriquant, Antonio Serra y Sobrino, apparaît aux extrémités du bifeuillet, son centre étant occupé par le blason du papetier avec une croix de Malte.

Autant dans le cas présent que dans celui du Breviario sunní précédent, on pourrait penser qu'il s' agit de copies réalisées pour un usage personnel, avec une fin érudite ou académique.

\subsection{Manuscrit de magie. Tunis (?), seconde moitié du XVII ${ }^{e}$ s./début du $X V I I I^{e 110}$}

De même que les deux derniers exemples (\$4.2 et $§ 4.3)$ ont été copiés en aljamia en caractères arabes par un Européen à une époque tardive, le manuscrit

\footnotetext{
106 Madrid, BNE MSs/1585.

107 Madrid, BNE MSS/18585/41. Madrid, BNE MSs/18585/41. Villaverde mentionne ces feuillets en supposant qu' ils ont pu venir de la bibliothèque de Gayangos (Villaverde, «Los manuscritos», p. 98).

108 «A diferencia de las filigranas de los papeles hechos a mano, las realizadas industrialmente podían durar un período superior a los dos años de las filigranas de molde». Hidalgo, «Interpretación material», p. 8 o.

109 «Es en el que están realizados la mayoría de estos textos manuscritos 'pasados a limpio'», ibidem, p. 93 .

110 Manuscrit décrit dans Martínez de Castilla, «A la búsqueda».
} 
évoqué ici a été copié dans une ville ottomane - probablement Tunis-111 à partir de la seconde moitié du XvII ${ }^{\mathrm{e}} \mathrm{s}$. Bien que le manuscrit soit transcrit en caractères maghrébins, une série d'indices indique qu'il est passé dans la partie orientale du monde musulman (s'il n'a pas été copié là-bas). Deux types de papier y sont utilisés, l'un comportant des filigranes dits Tre lune dont la production débute vers $1640^{112}$.

De façon exceptionnelle par rapport à ce qui se passe dans la production aljamiada, le copiste du manuscrit était plus familier avec l'arabe qu' avec l'aljamia. De manière surprenante, il n'emploie pas le système de translittération connu et employé par les Mudéjares et les Morisques, ce alors qu'une bonne partie de ses contenus sont présents dans un manuscrit d'Almonacid $(\S 1.3 \cdot 1)^{113}$.

\section{$5 \quad$ Conclusion}

Pendant le Moyen Âge, les Mudéjares ont développé un système de translittération du castillan en cacactères arabes qui était stabilisé en 1462. Ce système a été adopté par les Morisques: c' est celui qui, à l'époque moderne, est connu sous le nom d' «aljamia» et qui est resté en usage jusqu' au début du XVII ${ }^{\mathrm{e}} \mathrm{s}$., moment de l'expulsion définitive des Morisques. Les textes qui emploient ce type d'écriture ont été élaborés et copiés dans les royaumes d'Aragon et de Castille, et de manière exceptionnelle en Afrique du Nord - dans ce cas par des Morisques expulsés qui y avaient trouvé refuge. Cette production était principalement destinée à un usage interne et visait à préserver et à diffuser la culture et la religion musulmane au sein des communautés mudéjares et morisques, mais pouvait en même temps être source de divertissement et contribuer à résoudre des problèmes quotidiens.

Si tel est bien le périmètre de cette production généralement qualifiée d'aljamiada, le phénomène est bien plus complexe et tant l'émetteur, le récepteur, le message, le code le canal et le contexte doivent être pris en compte afin de pouvoir réellement comprendre ces textes.

Tous les textes écrits par des Mudéjares et des Morisques n'emploient pas le castillan en caractères arabes - l' alphabet latin et la langue arabe interviennent également. Tous les textes castillans en caractères arabes n'ont pas été écrits

\footnotetext{
111 Harvey, «Magic and Popular Medicine».

112 Velkov et Andreev, Filigranes.

113 Harvey, «Magic and Popular Medicine».
} 
par des Mudéjares et des Morisques et n'ont pas été faits pour circuler dans ces communautés. De même, tous les textes écrits par des Mudéjares et des Morisques ne présentent pas un même état de la langue castillane: les textes traduits de l'arabe présentent un degré plus élevé d'arabisme et d' archaïsme par rapport aux compositions plus récentes ou empruntées à la tradition occidentale, toutes rédigées dans l'espagnol standard de l'époque. Dans les deux cas, l'alphabet employé peut être soit arabe, soit latin, de même qu'il existe au sein de ces communautés des textes en arabe écrits en caractères arabes ou latins (ce dernier cas étant très exceptionnel).

Tous les textes castillans en caractères arabes n'ont pas été produits entre le $\mathrm{XV}^{\mathrm{e}}$ s. et le début du XVII ${ }^{\mathrm{e}}$; ils n'ont pas davantage été tous produits en Aragon et en Castille. Il s' en trouve en effet qui ont été copiés auX XVIII ${ }^{\mathrm{e}}$, XIX ${ }^{\mathrm{e}}$ et XX ${ }^{\mathrm{e}} \mathrm{s}$. dans d'autres lieux et dans de nombreux cas il s' agit des mêmes que ceux qui ont été traduits ou copiés au XVI ${ }^{\mathrm{e}} \mathrm{s}$.

Les destinataires de ces textes n' étaient pas toujours des membres des communautés mudéjares et morisques, mais parfois des copistes qui en étaient issus ont produit des manuscrits pour des chrétiens; de même, les Morisques aussi ont copié des textes qui avaient été produits par des chrétiens. Il est arrivé également que des œuvres composées par des Mudéjares ou des Morisques soient transmises par des copistes étrangers à ces communautés, pour un usage en dehors de ces dernières, tant à la même époque que par la suite.

Pour chacune des situations présentées, les interprétations changent, bien évidemment. Un récit musulman racontée à un public mudéjare (avant la conversion forcée), morisque (après la conversion forcée), inquisitorial, ottoman de la fin du XVIII ${ }^{e}$ s. ou espagnol (sans affiliation religieuse connue) de la fin du XIX ${ }^{\mathrm{e}}$ s. ne représente pas la même chose. Le support, l'écriture, la mise en page, le décor, le message, l' émetteur, le récepteur et le contexte sont différents; de ce fait, le sens du texte, même s' il reste le même dans tous les cas, sera affecté.

Si nous voulons parler de la production et de la transmission des textes d'un point de vue culturel dans les communautés mudéjares et morisques des $\mathrm{XV}^{\mathrm{e}}$ $\mathrm{XVII}^{\mathrm{e}} \mathrm{S}$., nous devrons élargir notre champ de vision à la production: d' un côté, nous pouvons réduire les langues employées par ces communautés au castillan en caractères arabes, mais il faudra aussi tenir compte du reste des langues qu' ils employaient, spécialement l' arabe, mais aussi le latin ou d' autres langues vernaculaires. D'un autre côté, nous ne pourrons pas nous limiter à n' analyser que les manuscrits provenant d'un milieu musulman et traitant de thèmes qui sont en rapport avec lui; il faudra également prendre en considération ceux qui ont été produits dans l' Espagne chrétienne et juive et qui ont été copiés ou ont simplement circulé dans ces communautés. Enfin, il sera nécessaire de tenir compte de l'emploi, à la même époque, de ces manuscrits par d' autres destina- 
taires, en dehors des communautés mudéjares et morisques proprement dites.

Si nous voulions parler du concept d' «aljamia» comme phénomène linguistique synonyme de «castillan en caractères arabes », nous devrions prendre du recul par rapport à l'époque et la culture mudéjares et morisques pour approcher n'importe quel texte castillan dans lequel cet alphabet est employé indépendamment de la période et du lieu dans lequel il a été produit ainsi que de l'usage qui en a été fait dans les différents contextes correspondants. En ce qui concerne les textes castillans en caractères arabes produits dans l'Espagne médiévale et moderne, il faudrait ignorer, ou, si c'est possible, résoudre deux problèmes pour parvenir à une définition claire: d'une part, le fait que l'on puisse trouver les mêmes textes écrits dans deux graphies différentes, et l' autre que le castillan attesté dans les manuscrits connaisse deux états de la langue différents selon l' origine du texte.

\section{Remerciements}

Cet article a été rédigé dans le cadre du project ERC 670628, SICLE.

The research leading to these results has received funding from the European Research Council under the European Union Funding for Research and Innovation (Horizon 2O2O) E RC Grant Agreement number 670628, SICLE (2O162021).

\section{Bibliographie}

Barceló, C., Un tratado catalán de derecho islámico. El Llibre de la çunna e xara dels moros, Cordue: Université, 1989.

Barceló, C. et A. Labarta, Archivos moriscos. Textos árabes de la minoría islámica valenciana 1401-16o8, Valence: Université, 2009.

Bernabé Pons, L., «Interferencias entre el árabe y el romance en los textos coránicos aljamiados », Lenguas en contacto: el testimonio escrito, éd. P. Bádenas de la Peña et al., Madrid: csic, 2004, pp. 109-126.

Casassas, X., «La literatura aljamiado-morisca en el marco de la literatura islámica española: siglos XIII-XVII (una variedad del castellano vinculada al hiero-sprachbund islámico)», Los moriscosy su legado desde estay otras laderas, éd. F. Benlabbah et A. Chalkha, Rabat: Instituto de Estudios Hispano-Lusos, 2010, pp. 368-396.

Castaño, J., «Nuevos documentos hebraico-aljamiados de Aragón(1). Fragmentos de un registro contable de pagos de la aljama de Tarazona», Sefarad 62.2 (2004), pp. 315340. 
Castaño, J., «Ketubbot en-cubiertas: fuentes para el estudio del matrimonio judío en Jaca y los Almosnino », Sefarad 69.1 (2009), pp. 43-73.

Castilla, N. de, El manuscrito aljamiado T19 de la Real Academia de la Historia. Edicion, estudio y glosario, Madrid: UCM, 2005.

Castilla, N. de, «Anduve mirando si parecía por allí algún morisco aljamiado », De Cervantes y el islam, éd. N. de Castilla et R. Gil, Madrid: SECC, 2006, pp. 235-246.

Castilla, N. de, «Corán de Salónica», dans Memoria de los moriscos, éd. A. Mateos et J.C. Villaverde, Madrid: SECC/BNE, 2O10, p. 228.

Castilla, N. de, «El libro manuscrito entre los moriscos», dans Memoria de los moriscos, éd. A. Mateos et J.C. Villaverde, Madrid: SECC/BNE, 2010, pp. 73-80.

Castilla, N. de, Una biblioteca morisca entre dos tapas, Saragosse: IEIOP, 2010.

Castilla, N. de, «Qur'anic Manuscripts from Late Muslim Spain», Journal of Qur'anic Studies 16.2 (2014): 89-138.

Castilla, N. de, «The Teaching and Learning of Arabic in Salamanca in the Early Modern Period», Learning and Teachings of Arabic in Early Modern Europe, éd. J. Loop, A. Hamilton et Ch. Burnett, Leiden: Brill, 2017, pp. 163-188.

Castilla, N. de et T. Dadson (éds.), L.P. Harvey. Islamic Culture in Spain to 1614. Essays and Studies, Oxford: Legenda, 2019.

Castilla, N. de, «A la búsqueda de manucritos moriscos perdidos. Nuevos testimonios aljamiados », Circulaciones mudéjares y moriscas, éd. A. Echevarría, A. Kadri et Y. Moreno, Madrid: cSIC, 2018, pp. 297-346.

Castilla, N. de, «Uses and written practices in Aljamiado manuscripts», Creating Standards: Interactions with Arabic Script in 12 Manuscript Cultures, éd. D. Bondarev, A. Gori et L. Souag, Berlin, Boston: De Gruyter, 2019, pp. 111-13o.

Castilla, N. de, Les manuscrits mudéjares et morisques en France, Rome (à venir).

Cervera Fras, M.J., Manuscritos moriscos aragoneses, Saragosse: IE IOP, 2010.

[CORDE] Corpus diacrónico del español www.rae.es.

Domínguez Ortiz, A. et B. Vincent, Historia de los moriscos. Vida y tragedia de una minoría, Madrid: Revista de Occidente, 1978.

Epalza, M., Los moriscos antes y después de la expulsión, Madrid: Mapfre, 1992.

Feliciano, M.J., «Breviario çunní de uso de la Inquisición», voir Memoria de los moriscos, p. 172.

Fernández y González, F., Estado social y político de los mudéjares de Castilla, Madrid: [Real Academia de la Historia], 1866 [réimprimé en Madrid: Hiperión, 1985].

Galmés de Fuentes, Á., «La lengua española de la literatura aljamiado-morisca como expresion de una minoría religiosa», Revista de la Sociedad Española de Lingüística 16.1 (1986), pp. 21-38.

Galmés de Fuentes, Á. (éd), Dichos de los siete sabios de Grecia, Madrid: Gredos, 1991.

Galmés de Fuentes, Á., Los manuscritos aljamiado-moriscos de la Biblioteca de la Real 
Academia de la Historia (Legado Pascual de Gayangos), Madrid: Real Academia de la Historia, 1998.

Galmés de Fuentes, Á. (éd), Tratado de los dos caminos: (ms. s2 de la Colección Gayangos, Biblioteca de la Real Academia de Historia), Madrid: UCM/U. Oviedo, 2005.

Galmés de Fuentes, Á. (éd), Historia de los amores de París y Viana, Madrid: Gredos, 1970.

García-Arenal, M., « Ríos y caminos moriscos. El islam tardío español», Revista de Libros 134 (2008), www.revistadelibros.com.

García-Arenal, M. et F. Rodríguez Mediano, Un oriente español. Los moriscos y el Sacromonte en tiempos de Contrarreforma, Madrid: Marcial Pons, 2010.

Gómez Renau, M., «La lengua aljamiada y su literatura: una variante islámica del español», Castilla. Estudios de literatura 25 (2000), pp. 71-83.

Harvey, L.P., «Un manuscrito aljamiado en la Biblioteca de la Universidad de Cambridge», Al-Andalus 23.1 (1958), pp. 49-74; dans Islamic Culture in Spain to 1614: Essays and Studies. L.P. Harvey, éd. N. de Castilla et T. Dadson, Oxford:Legenda, 2019, pp. 184-20o.

Harvey, L.P., «El Mancebo de Arévalo y la literatura aljamiada», Actas del Coloquio Internacional sobre Literatura Aljamiada y Morisca, Madrid: 1978, pp. 21-42; dans Islamic Culture in Spain to 1614: Essays and Studies. L.P. Harvey, éd. N. de Castilla et T. Dadson, Oxford: Legenda, 2017, pp. 6-19.

Harvey, L.P., «Magic and Popular Medicine in an Aljamiado Manuscript, possibly of Tunisian Provenance, sold in London in 1993», Romania Arabica. Festschrift für Reinhold Kontzi 96 (1996), pp. 335-344, dans Islamic Culture in Spain to 1614: Essays and Studies. L.P. Harvey, éd. N. de Castilla et T. Dadson, Oxford: Legenda, 2017, pp. 218227.

Hegyi, O., «Una variante islámica del español: la literatura aljamiada», Homenaje a Álvaro Galmés de Fuentes, Madrid: Gredos, 1987, pp. 647-655.

Hernández, F.J., «En la prehistoria de la materia épica cidiana: el Cid no era el Cid», Revista de Filología Española 89 (2009), pp. 257-278.

Hidalgo Brinquis, C., «Interpretación material de los catálogos monumentales de España», El catálogo monumental de España (1900-1961): investigación, restauración y difusión, éd. Amalia López-Yarto, [Madrid]: MECyD, 2012, pp. 75-106.

Jiménez Moreno, A., «Formación, uso y dispersión de una pequeña biblioteca nobiliaria del siglo Xv: los libros de doña Leonor Pimentel, condesa de Plasencia », Literatura medieval y renacentista en España: líneas y pautas, Salamanca: SEMYR, 2012, pp. 655-663.

Labarta, A., «Oraciones cristianas aljamiadas en procesos inquisitoriales de moriscos valencianos», Boletín de la Real Academia de Buenas Letras de Barcelona 37 (19771978), pp. 177-197.

Labarta, A., Libro de dichos maravillosos: misceláneo morisco de magia y adivinación, Madrid: CSIC/ICMA, 1993. 
Labarta, A. et C. Barceló, «Latín y romance en oraciones cristianas halladas a moriscos valencianos», in Festschrift für Reinhold Kontzi, Tübingen: Narr, 1996, pp. 315321.

Liman, T., Edición crítica, estudio lingüístico y glosario delmanuscrito aljamiado-morisco núm. 6016 de la Biblioteca Nacional de Madrid, thèse de doctorat inédite, Madrid: UCM, 1995 .

López-Baralt, L. et A. Irizarry, «Dos itinerarios moriscos secretos del siglo XVI: el ms. 774 París y el ms. T-16 RAH », Homenaje a Álvaro Galmés de Fuentes, Madrid: Gredos, 1985, pp. 547-582.

López-Morillas, C., «The Genealogy of Spanish Qur’an», Journal of Islamic Studies 17.3 (2006), pp. 255-294.

López-Morillas, C., El Corán de Toledo. Edición y estudio del manuscrito 235 de la Biblioteca de Castilla-La Mancha, Gijón: Trea, 2011.

Martínez de Castilla Muñoz, N., voir "Castilla, N. de".

Marquer, J., Propagande politique et Islam d'Occident sous le règne de Pierre Ier de Castille (1350-1369), thèse de doctorat inédite, Paris: Paris IV-Sorbonne, 2014.

Medina Morales, F., «Las formas nominales de tratamiento en el Siglo de Oro. Aproximación socio-lingüística», Memoria de la palabra. Actas VI Congreso de la AISo, éd. M.L. Lobato et F. Domínguez Matito, Frankfurt am Main/Madrid: Iberoamericana/Vervuert, 2004, pp. 1329-1341.

Menéndez Pidal, R. (éd), Poema de Yúçuf. Materiales para su estudio, Grenade: Université, $195^{2}$.

Montaner Frutos, A., «La aljamía: una voz islámica en Aragón», Jornadas sobre la variación lingüistica en Aragón a través de los textos, Saragosse: Institución «Fernando el Católico», 2003, pp. 99-204.

Montaner Frutos, A., «La literatura aljamiada», dans Memoria de los moriscos, éd. A. Mateos et J.C. Villaverde, Madrid: SECC/BNE, 2O1O, pp. 45-55.

Montaner Frutos, A., «La Historia Roderici y el archivo cidiano: Cuestiones filológicas, diplomáticas, jurídicas e historiográficas », e-Legal History Review 12 (2011), pp. 1-62.

Ribera, J. et M. Asín (éds), Manuscritos árabes y aljamiados de la Biblioteca de la Junta, Madrid: JAE, 1912.

Sánchez Álvarez, M., El manuscrito misceláneo 774 de la Biblioteca Nacional de París, Madrid: Gredos, 1982.

Sánchez Álvarez, M., «Sobre la variante islámica del español», Actas del I Congreso Internacional de Historia de la Lengua Española II, Madrid: ArcoLibros, 1988, pp. 1355-1364.

Suárez García, R. (éd), El compendio islámico de Mohanmad de Vera. Un tratado morisco tardío, Oviedo: Université, 2016.

Suárez Piñera, R., Publicacióny estudios de los pasajes aljamiados del ms. 1163 de la Biblioteca Nacional de París, thèse de doctorat inédite, Oviedo: Université, 1973. 
Valencia, R., «Las inscripciones árabes en el arte mudéjar », Mudéjar, el legado andalusí en la cultura española, Saragosse: 2010, pp. 293-305.

Ubieto, A., Colección diplomática de Pedro I de Aragón y Navarra, Saragosse: CSIC, 1951. Velkov, A. et S. Andreev, Filigranes dans les documents ottomans. I Trois croissants, Sofia: 1983.

Villaverde, J.C., «Los manuscritos aljamiado-moriscos: hallazgos, colecciones, inventarios y otras noticias », dans Memoria de los moriscos, éd. A. Mateos et J.C. Villaverde, Madrid: SECC/BNE, 2O1O, pp. 91-128.

Wiegers, G., Islamic Literature in Spanish and Aljamiado. Yça de Segovia (f. 1450), His Antecedents and Successors, Leiden: Brill, 1994.

Wiegers, G., «Datation et localisation des codices espagnols écrits en caractères arabes (aljamiado): Problèmes et perspectives», Le manuscrit arabe et la codicologie, éd. A. Binebine, Rabat: 1994, pp. 21-30.

Wiegers, G., «Breviario Çunní, de Iça de Gebir», voir Memoria de los moriscos, p. 132.

Zanón, J., «Los estudios de lengua árabe entre los moriscos aragoneses a través de los manuscritos de la Junta», Sharq al-Andalus 12 (1995), pp. 363-374. 